\title{
Hierarchical micro/nano structures for super-hydrophobic surfaces and super-lyophobic surface against liquid metal
}

Youngsam Yoon ${ }^{*}$, Daeyoung Kim and Jeong-Bong Lee

\begin{abstract}
Non-wetting super-hydrophobic or super-lyophobic surfaces are of great interest in a variety of applications. Natural water repelling surfaces show micro-/nano- combined hierarchical structure which shows extremely low wettability and self-cleaning characteristics. Inspired by such natural wonders, there have been tremendous efforts to create artificial non-wetting super-hydrophobic or super-lyophobic surfaces. In this paper, recent progress in artificial super-hydrophobic surfaces based on hierarchical micro-/nano- dual-scale structure is reviewed. In addition, application of hierarchical micro-/nano- dual-scale structure as super-lyophobic surfaces against gallium-based liquid metal alloy is also reviewed.
\end{abstract}

Keywords: Super-hydrophobic; Super-lyophobic; Lotus effect; Self-cleaning; Hierarchical structure; Liquid metal; Galinstan ${ }^{\circledast}$

\section{Review}

Natural water repelling surfaces, such as lotus leaves, butterfly wings, and shark scales exhibit high water contact angle in excess of approximately $150^{\circ}$ [1-3]. Surfaces with water contact angles greater than $150^{\circ}$ is called super-hydrophobic. It has been reported that such superhydrophobicity stems from a combination of hierarchical micro-/nano-scale surface texturing and surface chemical composition [4]. Combination of micro- and nano-scale structure is crucial as water droplet on these hierarchical structures may touch on the apex of the nano-scale structures without fully sitting on the surface because air pockets fill in the vicinity of nano-scale structures beneath the droplet resulting in low contact area of water droplet, high contact angle, low contact hysteresis, and low adhesive force [5].

Mimicking such natural wonders and creating artificial super-hydrophobic surfaces on various solid substrates has been of great interest over the past few decades and there have been considerable amount of reported literatures on the topic. Although a variety of materials and technologies were studied to create artificial hierarchical

\footnotetext{
*Correspondence: yyoon4@gmail.com

* Correspondence: yyoon4@gmail.com
Department of Electrical Engineering, The University of Texas at Dallas, Richardson, TX, USA
}

micro- /nano-scale surface textures for super-hydrophobicity, many of the studies are based on materials that are solid and optically opaque in visible wavelengths. Superhydrophobic surfaces with high optical transmission in visible wavelengths have considerable potential to be used in a variety of self-cleaning surface applications such as optical component, lens, displays, electronic equipment, automobile glass, anti-fog consumer glass, solar panels, among others. In order to maintain high optical transparency, it is critical that structural roughness on the artificial lotus effect surface should be much smaller than the wavelength of transmitted light [6].

Along with super-hydrophobic surfaces, there have been great interests in creating anti-wetting surfaces against oils [7] as well as microfluidic platforms for manipulating gallium-based non-toxic liquid metals [8]. With the phenomenal advance of artificial super-hydrophobic surfaces, hierarchical micro-/nano-scale surfaces may provide starting platforms for study on creating super-oleophobic surfaces and super-lyophobic surfaces against liquid metals.

This review discusses recent research progress on hierarchical micro-/nano-scale surfaces as artificial superhydrophobic surfaces as well as super-lyophobic surfaces against gallium-based liquid metal alloys. 


\section{Theoretical backgrounds}

\section{Definition of lyophobic/lyophilic}

The definition of 'lyophobic/lyophilic' is the physical property of a molecule that is repelled from or attracted to a mass of liquid. Therefore, the 'lyophobic or lyophilic' should be used with indication of target liquid except hydrophobic/hydrophilic or oleophobic/oleophilic that implies target liquid is water or oil, respectively. The 'lyophobic/lyophilic' is the superordinate concept to hydrophobic/hydrophilic.

In order to characterize lyophobicity (either lyophobic or lyophilic), static and dynamic contact angles are important parameters. Static contact angles can be measured by using a contact angle goniometer which can capture the profile of a liquid droplet on a solid substrate. Ideal static contact angle depends on the surface tensions of liquid (liquid/gas interfacial energy) and solid (solid/gas interfacial energy) and liquid/solid interactions. As shown in Figure 1a, the static contact angle is defined as the angle formed by the intersection of the liquid-solid interface and the liquid-gas interface.

Generally, when the contact angle is smaller than $90^{\circ}$, the solid surface is considered to be 'lyophilic' which means wetting of liquid on the surface is favorable. When the contact angle is larger than $90^{\circ}$, the solid surface is considered 'lyophobic' which indicates the liquid will minimize its contact on the surface and form a compact liquid droplet. When the contact angle is greater than $150^{\circ}$, the solid surface is considered to be 'super-lyophobic'. And when the contact angle is almost $0^{\circ}$, the surface is considered to be 'super-lyophilic' [9].

\section{Surface tension}

Ideally, as explained, the contact angle of liquid on the solid is determined by the surface tension of liquid, surface energy of solid, and their interaction. In a pure liquid, as shown in Figure 2, molecules in the bulk of liquid have interactions with all neighboring molecules so that the net force should be zero. The interactions are mostly originated from Van der Waals forces for non-polar liquids and hydrogen bonds for polar liquids such as water. On the other hand, molecules exposed at the surface do not have interactions on all sides of them to provide a balanced net force, resulting in the local asymmetry of interactions. The resulting dissymmetry in the interactions causes pulling internal force which is called as the surface tension. Therefore, surface tension of the liquid changes its geometry to minimize this energy defect, forming a spherical shape. Generally, for a solid substrate, the 'surface energy' is used to have equivalent meaning of surface tension for a liquid.

\section{Wetting models}

As shown in Figure 1a, Thomas Young described the contact angle of a liquid droplet on an ideal solid substrate [9]. It is determined by the thermodynamic equilibrium of surface tensions of three interfaces under the droplet:

$$
\cos \theta=\frac{\gamma_{S G}-\gamma_{S L}}{\gamma_{L G}}
$$

where $\theta$ is contact angle and $\gamma_{S G}, \gamma_{S L}$, and $\gamma_{L G}$ are surface tension of solid-gas, solid-liquid and liquid-gas interfaces, respectively. Equation (1) is usually referred as Young's equation. It should be noted that Young's equation only works with flat homogeneous surface. So, recent advances in artificial super-hydrophobic surfaces with non-planar geometry should be modeled by modified equations other than Young's equation. On roughened surfaces, there are mainly two different equilibrium states: Wenzel and Cassie state. As shown in Figure 1b, when a liquid can fully wet the surface texture, the thermodynamic equilibrium contact angle of a liquid droplet is described by the Wenzel model [10] which is given by:

$$
\cos \theta_{W}=r \cos \theta
$$

where $\theta_{w}$ is the apparent contact angle in Wenzel state, $\theta$ refers to Young's contact angle, and $r$ is a roughness factor, which is defined as the ratio of actual area of a rough surface to the flat, projected area. Droplets in this fullywetted Wenzel state typically display very high hysteresis because the contact line of the droplets becomes severely pinned on surface asperities. On the other hand, when a liquid cannot penetrate into the surface texture, the droplet forms a highly non-wetting regime known as a CassieBaxter state or simply Cassie state (Figure 1c). Because of a decrease in the effective contact area between a surface and a droplet by entrapped air, it can have low adhesive force. Therefore, compared to Wenzel state, the droplet in the Cassie state displays a very high contact angle as well
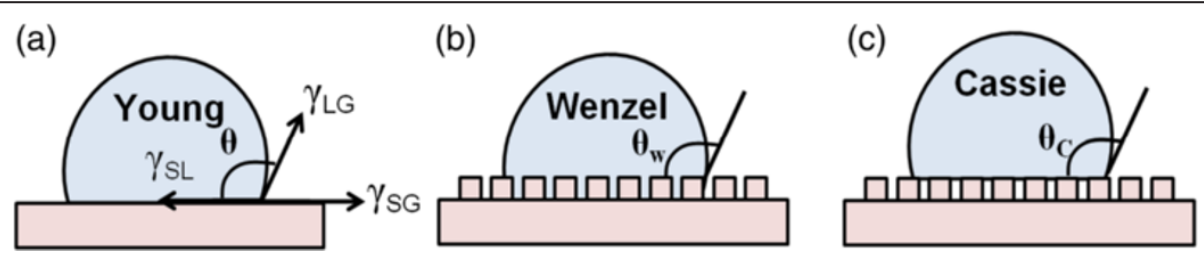

Figure 1 Schematic diagrams of different wetting regimes: (a) Young's model, (b) Wenzel model, and (c) Cassie-Baxter model. 


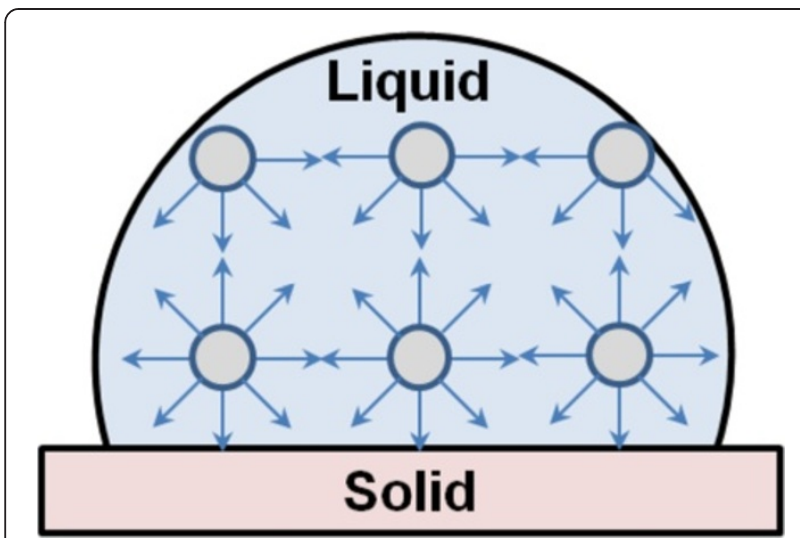

Figure 2 A schematic diagram of surface tension of liquid due to asymmetry of interactions at the surface of liquid.

as low hysteresis, resulting in easy roll-off characteristic due to its low adhesive force between the liquid droplet and the solid substrate. If $f_{s}$ is the fraction of the solid in contact with the liquid, the Cassie equation can be represented by equation (3) [11]:

$$
\cos \theta_{C}=f_{S}(1+\cos \theta)-1
$$

where $\theta_{C}$ is effective contact angle in Cassie state. This equation indicates the contact angle increases as the solid fraction decreases in Cassie state. Additionally, in order to have more accurate predictions of advancing and receding contact angles, the modified Cassie-Baxter relation which includes a local differential texture parameter was proposed [12].

\section{Contact angle hysteresis}

Along with static contact angle, in order to quantify the 'dynamic' wetting property of liquid on a solid surface (when the droplet is in the transitional motion), 'dynamic' contact angle is an important parameter. When a droplet is placed on an inclined surface, the droplet can experience gravitational force so that the shape of the droplet becomes asymmetric; on the downhill side, the droplet advances but on the uphill side, the droplet recedes. Therefore, the contact angle of the droplet on the downhill side is an advancing contact angle and that of the droplet on the uphill side is a receding contact angle. The difference between the advancing angle and the receding angle is called contact angle hysteresis. Due to the difference of advancing and receding contact angles, the droplet can stick to the surface against gravitational force. Additionally, the dynamic contact angle can be measured by changing the volume of liquid. The volume of liquid is increased or decreased until the droplet has a maximum or minimum contact angle without changing the surface area between the liquid and the solid substrate. The maximum and minimum contact angles are called the advancing and receding contact angle, respectively, and the advancing and receding contact angles are obtained from a series of images from a recorded video just before the contact line changes. When the advancing and receding contact angles of liquid on a solid substrate are close each other, which means the lower contact angle hysteresis, it indicates the substrate is lyophobic to the liquid [13].

\section{Super-hydrophobic surfaces}

\section{Super-hydrophobic surfaces in nature}

The hydrophobic property of nature's Nelumbo nucifera (lotus) has been observed by Barthlott and Neinhuis [14]. It was shown that water droplets placed on the apex of nanostructures combined with microstructures do not wet the surface as the hierarchical structure provides air pockets due to the dual-scale roughness morphology [15]. Surface of the lotus showed static contact angle and contact angle hysteresis approximately $164^{\circ}$ and $3^{\circ}$, respectively [16]. Such super-hydrophobicity of the natural lotus leaf surface enables self-cleaning of the leaf surface as the roll-off water droplets collect dusts in the path. As shown in SEM images (Figure 3), it clearly showed micro-scale elliptic protrusions with an average diameter of approximately $9.4 \mu \mathrm{m}$, pitch distance of $12 \mu \mathrm{m}$, and structural height of approximately $18 \mu \mathrm{m}$. In addition, surface of the micro-scale protrusions and bottom surface were thoroughly covered with random nano-scale textures. There are countless examples of natural super-hydrophobic surfaces in the plants such as taro (Colocasia esculenta), India canna (Canna generalis bailey), and rice leaf [17]. As shown in Figure 4, the large water contact angle of taro leaf, India canna, and rice leaf were $159 \pm 2^{\circ}, 165 \pm 2^{\circ}$, and $157 \pm 2^{\circ}$, respectively and there were homogeneous conformal structures on the surface in the range of few nanometer to micrometer which influence the wettability of surfaces. The sliding angle of taro leaf is $3^{\circ}$ and that of rice leaf is $4^{\circ}$ or $12^{\circ}$ depending on the direction of sliding of a droplet (parallel and perpendicular direction to surface papillae) due to anisotropic wetting property.

Surface morphology inducing water repelling characteristics of insect wings were also investigated and it was again found that surface of the insect wings have hierarchical micro-/nano-scale surfaces. Figure 5 shows water contact angle of various insect wings such as Homoptera Meimuna opalifera (Figure $5 \mathrm{a}, 165^{\circ}$ ), Orthoptera acrida cinerea (Figure $5 \mathrm{~b}, 151^{\circ}$ ), and Hymenopetra vespa dybowskii (Figure $5 \mathrm{c}, 125^{\circ}$ ) as well as SEM images of hierarchical structures which consist of micro-/nano-scale layers on the upper surfaces of insect wings [1,2]. From the nature's plant leafs and insect wings, it is clear that the hierarchical micro-/nano-scale surfaces are essential to demonstrate super-hydrophobicity. 

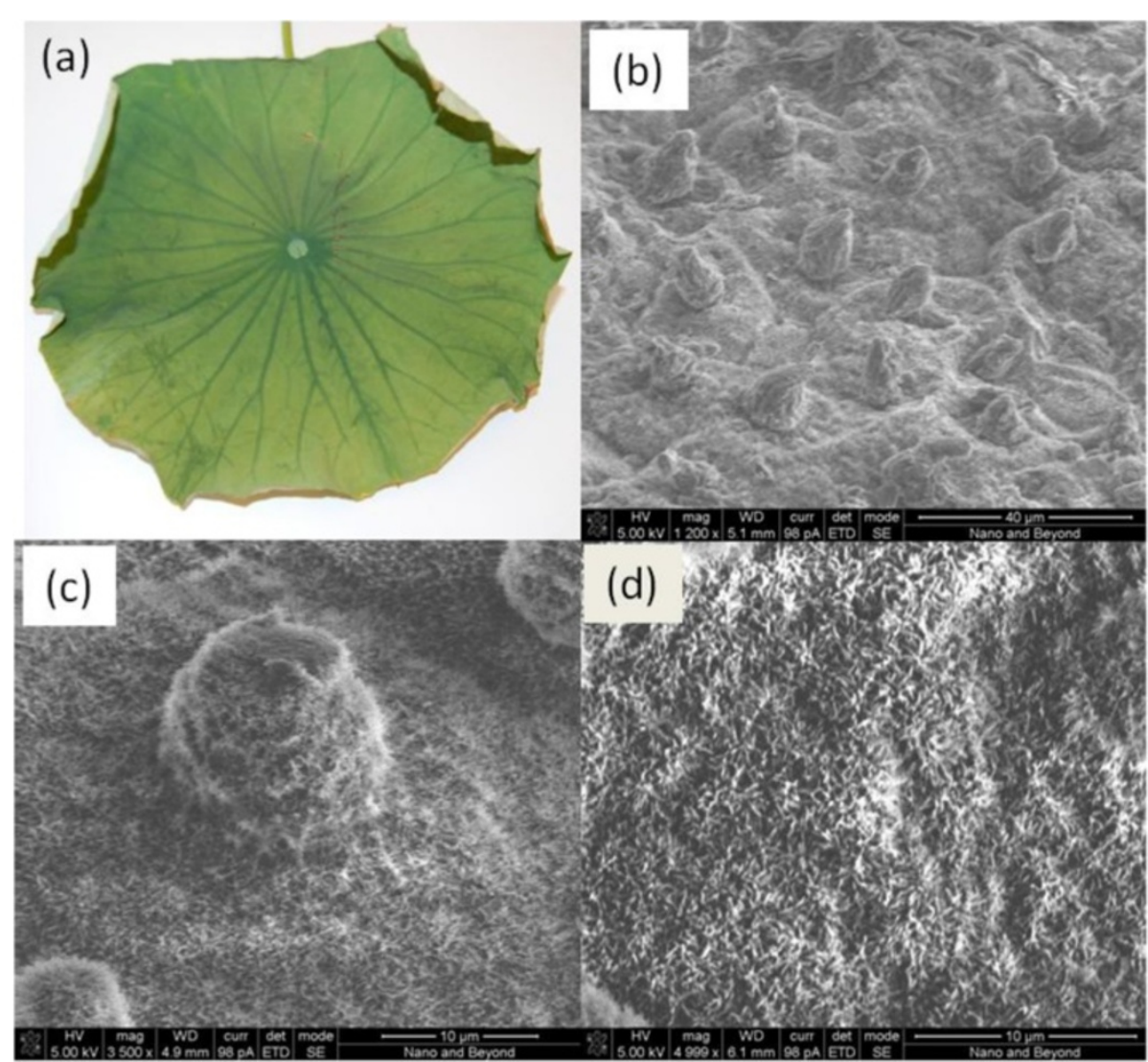

Figure 3 Natural lotus leaf: (a) an optical image and (b-d) SEM images.

\section{Artificial super-hydrophobic surfaces}

Understanding reasons for the super-hydrophobicity in various plant surfaces and insect wings among others in nature has allowed extensive researches in artificial superhydrophobic surfaces using various materials. A surface with combined characteristics of high water contact angles $\left(>150^{\circ}\right)$ with low sliding angles (tilted angle which allows free rolling of water droplets on a surface) typically smaller than $10^{\circ}$ is commonly known as self-cleaning surfaces. One of the motivations for the popularity in the
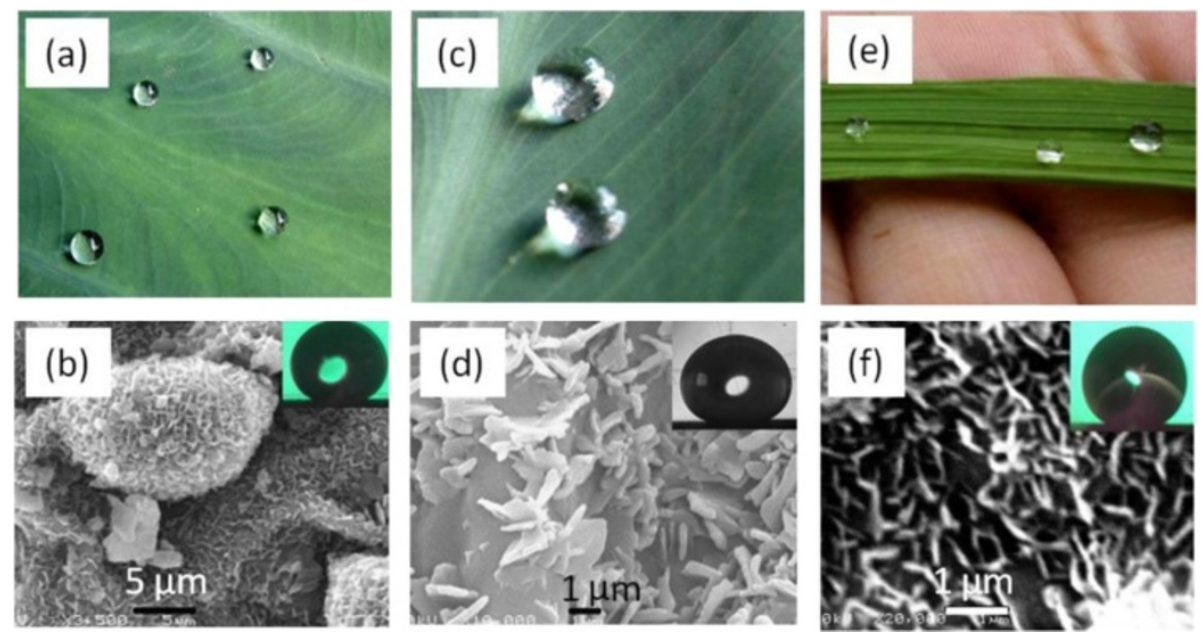

Figure 4 Various natural super-hydrophobic surfaces: (a) water droplets on a taro leaf, (b) SEM image of the taro leaf surface, (c) water droplets on an India canna leaf, (d) SEM image of the India canna leaf surface, (e) water droplet on a rice leaf, (f) SEM image of the rice leaf surface. With permission from Elsevier, Copyright 2007. 

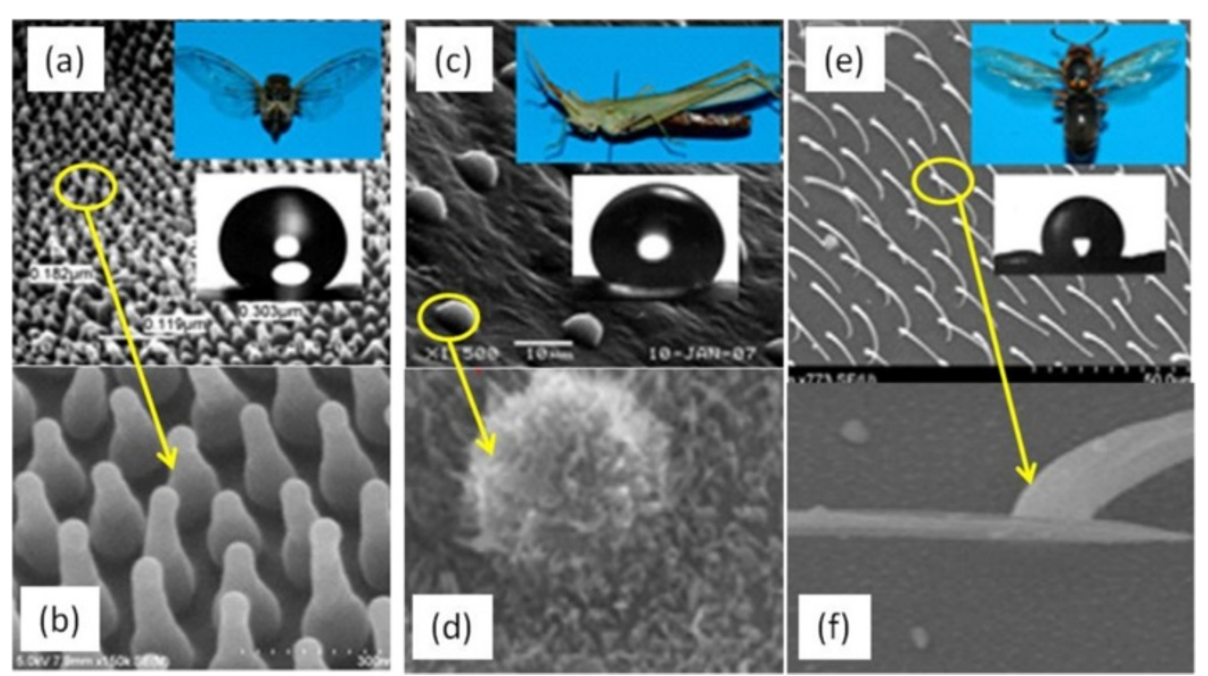

Figure 5 SEM images of the hierarchical micro-/nano-scale structures of the surface of the wings of various insects: (a, b) Homoptera Meimuna opalifera (Walker), (c, d) Orthoptera Acrida Cinerea cinerea (Thunberg), (e, f) Hymenoptera Vespa dybowskii (Andre). With permission from Elsevier, Copyright 2009.

research community on the super-hydrophobic surfaces is because of potential manufacturing of artificial selfcleaning surfaces. Inspired by natural super-hydrophobic surfaces, a wide variety of fabrication processes such as deep reactive ion etch (DRIE) process [18,19], electrodeposition [20-22], self-assembly [23], plasma treatment $[21,24]$, chemical vapor deposition $[22,25,26]$, and layer-by layer deposition [27] have been studied to fabricate artificial biomimetic super-hydrophobic hierarchical micro-/ nano-scale structures.

Among various artificial super-hydrophobic surfaces, optically transparent super-hydrophobic surfaces have great potential to be used in a variety of self-cleaning surface applications such as the prevention of the adhesion of dust and snow to window glasses, traffic indicators, goggles, and solar panels, etc. Optically transparent coatings, selfcleaning surfaces and anti-reflective coatings are active research topics targeted for the coating industry, consumer glass, optical component/lens manufactures, displays/ electronic equipment and aerospace. In order to make surfaces transparent to certain range of wavelengths of light, it is essential that the surface roughness of the artificial superhydrophobic surfaces should be much smaller than the wavelength of light [6]. This provides one of the key design criteria for the optically transparent self-cleaning surfaces which should have nano-scale roughness much smaller than wavelength of visible light $(400 \sim 700 \mathrm{~nm})$ while having micro-scale roughness for enhanced air-trapping.

Among various hierarchically textured super-hydrophobic surfaces, in order to introduce various materials and methods to fabricate them, we have studied four different super-hydrophobic surfaces: silica nanoparticles, carbon nanotube (CNT), PDMS, and SU-8 based superhydrophobic surfaces.

\section{Silica nanoparticles based super-hydrophobic thin film}

In order to realize artificial hierarchical micro-/nano- dualscale surface roughness, silica based raspberry-like nanoparticles were investigated. W. Ming et al. showed a superhydrophobic film from well-defined raspberry-like silica nanoparticles (Figure 6) using Stöber method [24,28]. The amine functionalized raspberry-like particles which were dispersed in ethanol were deposited on the epoxy film through the reaction between amine and epoxy at $75^{\circ} \mathrm{C}$. Therefore, loose particles could be flushed away with ethanol, and then only one layer of the raspberry-like particles covalently bonded to the epoxy based films. It showed a high advancing angle of $165 \pm 1^{\circ}$ along with a contact angle hysteresis of $\sim 2^{\circ}$, and a low sliding angle of $3 \pm 1^{\circ}$ against $10 \mu \mathrm{L}$ water droplet on the surface. Although, this approach demonstrated a super-hydrophobic surface, it required a series of relatively complex process such as the preparation of amino-functionalized nanoparticles, epoxyfunctionalized micro-particles, and a long reaction and separation procedure.

Additionally, with a relatively simple procedure, Qian et al. also developed the hierarchical dual-scaled raspberrylike particles by introducing poly acrylic acid (PAA) - functionalized polystyrene (PS) particles into hydrolysis reaction of tetraethoxysilane (TEOS). As shown in Figure 7, PAAfunctionalized PS was mixed with an ethanol solution containing TEOS and ammonia was added to catalyze the hydrolysis reaction of TEOS to form the silica nanoparticles around the PS. Then, raspberry particles were easily made 


\section{(a)}

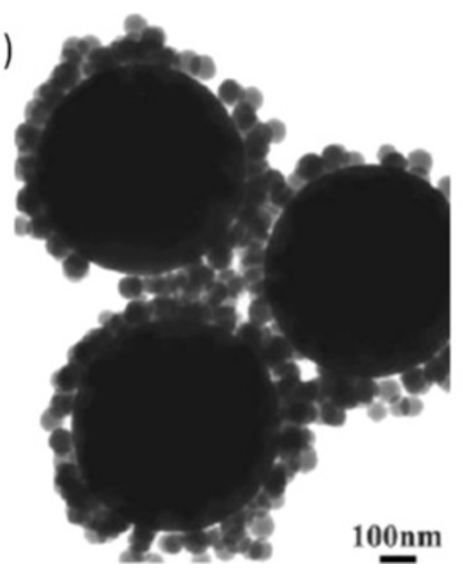

(b)

Figure 6 Artificial super-hydrophobic surfaces. (a) TEM image of raspberrylike silica particles and (b) an optical image of water droplet placed on the surface.

after centrifugation, and it was deposited on the glass substrate for hierarchical structured morphology. After modification of the surface with dodecyltrichlorosilane, super-hydrophobic surfaces were produced with a high contact angle of $162.1^{\circ}$ (Figure 8) [29].

\section{Carbon nanotube based super-hydrophobic thin film}

Based on utilizing nanoparticles, super-hydrophobic thin film can be realized by achieving artificial hierarchical dualscale surface roughness. Li et al. reported aligned CNT (ACNT) films which had perpendicular nanotubes against the substrate showed super-hydrophobic property [26]. Due to its structural property, it can have large fraction of air inducing a low contact area. The grown ACNT films showed contact angle of $158.5 \pm 1.5^{\circ}$ and it was increased to $171 \pm 0.5^{\circ}$ by immersion in a methanolic solution of hydrolyzed fluoroalkylsilane. The super-hydrophobic property of ACNT was enhanced by having low surface energy materials. Similarly, Lau et al. also reported that carbon nanotube forest coated with polytetrafluoroethylene (PTFE) showed the stable super-hydrophobic property [30] as shown in Figure 9. The grown CNT forest showed initial static contact angle of $161^{\circ}$ but the water droplets were not stable and permeated into the CNT forest voids after a few minutes. However, by depositing PTFE layer onto the CNT forest, the advancing and receding contact angles were measured to be $170^{\circ}$ and $160^{\circ}$ indicating the stable superhydrophobic property. In addition, Jung and Bhushan

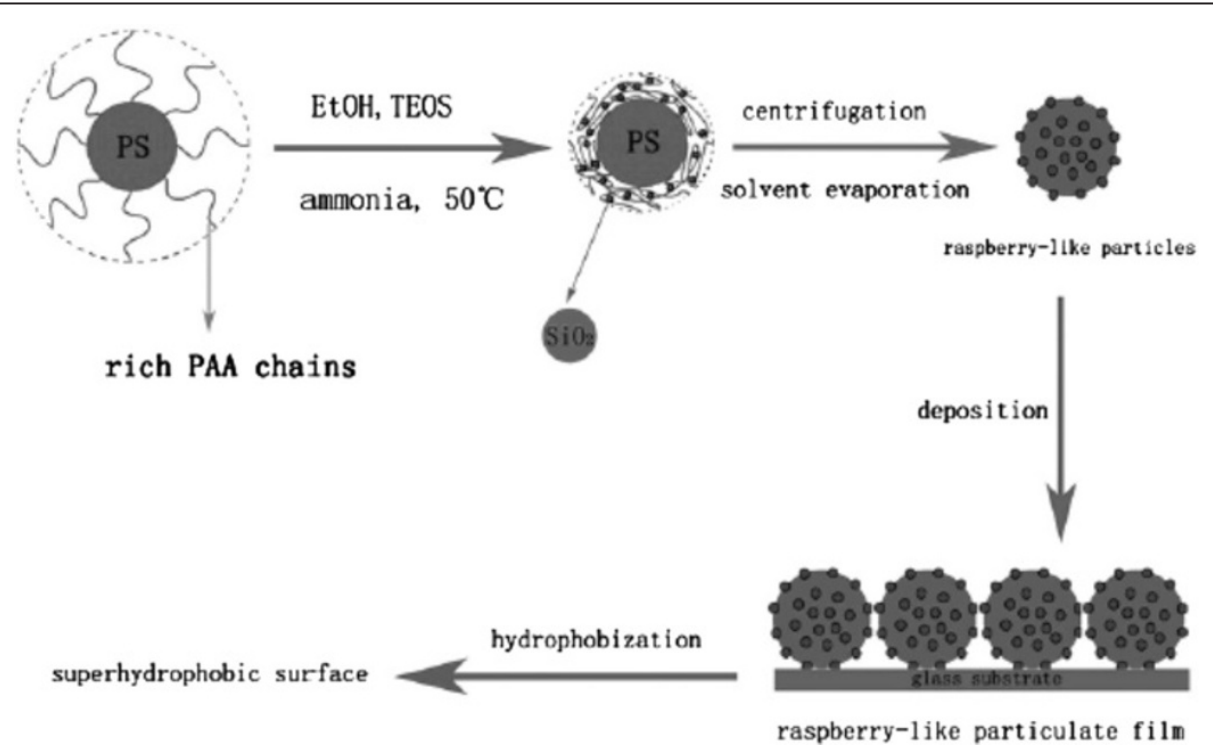

Figure 7 Schematic diagram of process for preparation of raspberry-like particulate films. With permission from Journal of Materials Chemistry, Copyright 2009. 


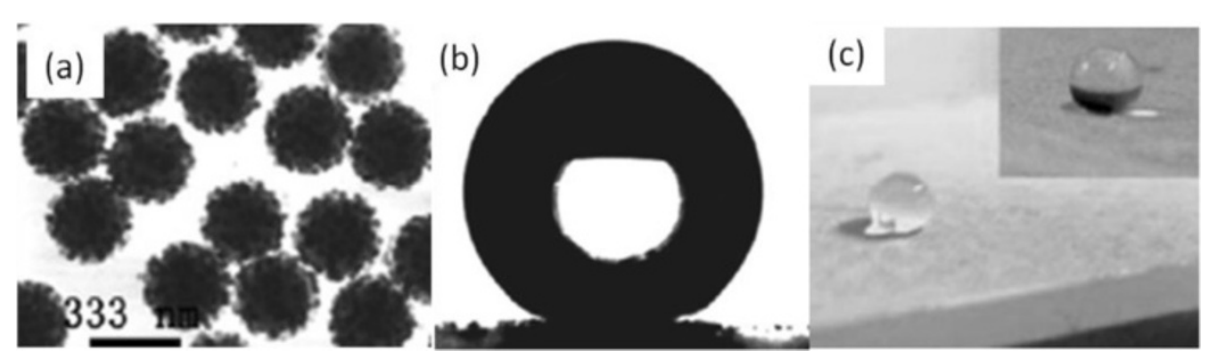

Figure 8 The raspberry like silica nanoparticles: (a) TEM image, (b) Contact angle, and (c) $10 \mu \mathrm{L}$ water droplet on the surface. The scale bar of (a) indicates $333 \mathrm{~nm}$. With permission from Journal of Materials Chemistry, Copyright 2009.

reported that the CNTs based hierarchical structure created by spray method and showed a high static contact angle of $170^{\circ}$ and a low contact angle hysteresis of $2^{\circ}$ [31]. The CNT-based hierarchical dual-scale structure could maintain its super-hydrophobic property even after exposing to water falling on it with a pressure of $10 \mathrm{kPa}$ for 24 hours. The static contact angle was remained greater than $150^{\circ}$ and the contact angle hysteresis was maintained less than $15^{\circ}$.

\section{SU-8 based super-hydrophobic thin film}

SU-8, one of the most popular materials used in the field of microelectromechanical systems (MEMS), shows a water contact angle of $73.1 \pm 2.8^{\circ}$ and surface energy of $45.5 \pm 0.3 \mathrm{~mJ} / \mathrm{m}^{2}$ [32]. Although the material itself does not show hydrophobicity, SU-8 is one of the great materials to create super-hydrophobic thin film because it is readily fabricated in high aspect ratio microstructures to (a)

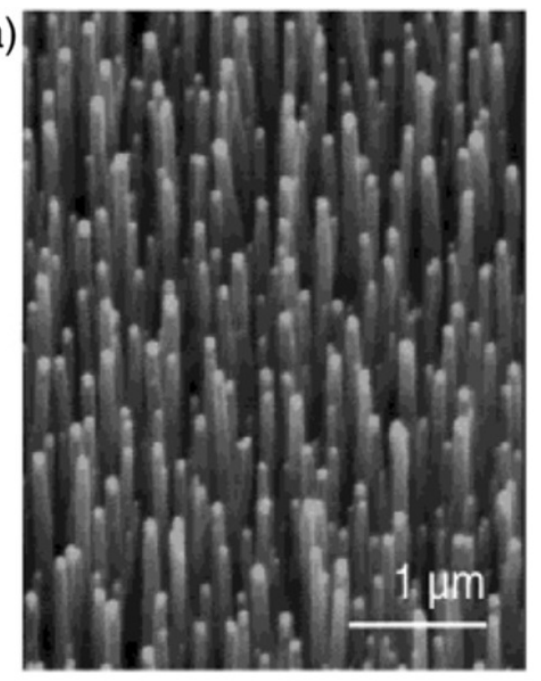

(b)

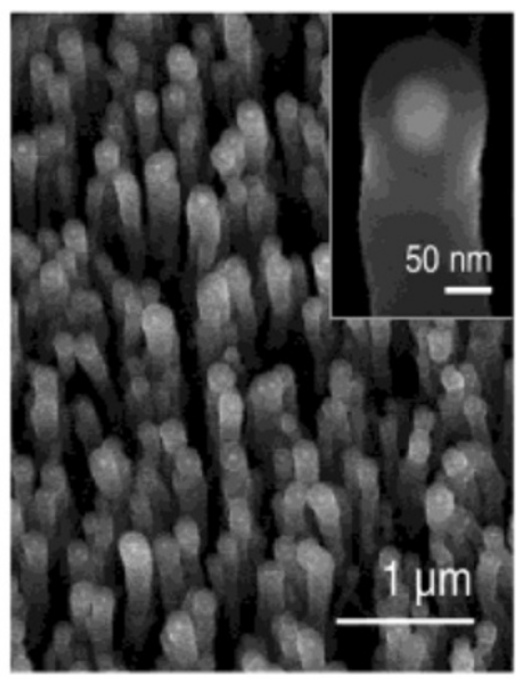

(c)

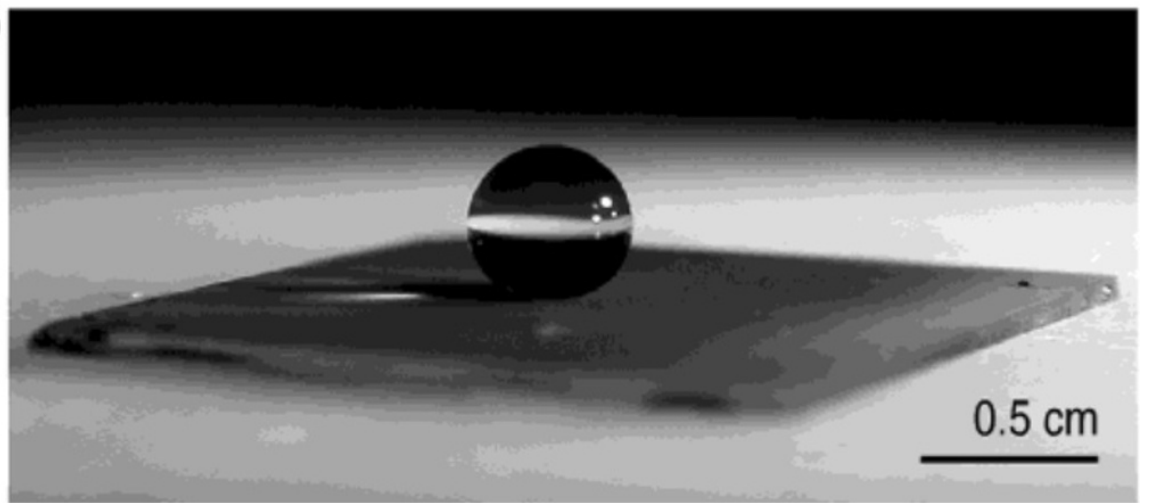

Figure 9 SEM images of carbon nanotube forests. (a) As-grown forest prepared by PECVD with nanotube diameter of $50 \mathrm{~nm}$ and a height of $2 \mu \mathrm{m}$, (b) PTFE-coated forest after HFCVD treatment, and (c) an essentially spherical water droplet suspended on the PTFE-coated forest. Reprinted with permission from American Chemical Society. Copyright (2003). 
have roughened surface which can be tuned its wetting property. In order to realize the property, it is essential to combine artificial nano-scale textures with the micropatterned SU-8, forming hierarchical micro-/nano-scale structured surface. Recently, there have been a few efforts to create artificial super-hydrophobic thin film using SU-8 as surveyed below.

Hong et al. prepared a direct mixture of polytetrafluoroethylene (PTFE) nanoparticles into SU-8, spin-coated the PTFE-SU-8 nano-composite on transparent substrates such as glass and polymers, and photo defined microscale patterns with a minimum feature size of $50 \mu \mathrm{m}$ [33]. This film showed water contact angle of $150^{\circ}$, which is significant improvement in hydrophobicity from normally not so hydrophobic SU-8 flat surface. This is attributed to the effect of formation of hierarchical roughness on the surface as well as reduction of effective surface energy of the substrate. Although this result seemed good, optical transparency of this PTFE-SU-8 nano-composite film was only $31 \%$, prohibitively low in practical optical transparency applications. They also reported an alternative method in which the PTFE nanoparticles were spray coated and thermally immobilized onto the exposed SU-8 matrix. During the SU-8 developing process, PTFE nanoparticles sprayed onto the unexposed SU-8 layer were also removed. The PTFE-SU-8 film prepared by this alternative method showed a water contact angle of $165^{\circ} \sim 167^{\circ}$ and an optical transparency up to $80 \%$.

Marquez-Velasco et al. reported super-hydrophobic surfaces fabricated in SU-8 by having micro- and nano-scale topography [34]. In this work, SU-8 was patterned to have low aspect ratio cylindrical and square pillars with thickness of $45 \mu \mathrm{m}$ and $75 \mu \mathrm{m}$. Then, the SU-8 pillars were $\mathrm{O}_{2}$ plasma treated to roughen the SU-8 pillar surface to have $20 \mathrm{~nm}$ (30 sec. etch) or $2 \mu \mathrm{m}$ (5 min. etch) sub-pillars formed on the SU-8. It was shown that $2 \mu \mathrm{m}$ sub-pillars were too tall and readily clung together to lose its purpose (dual-scale topography). However, dual-scale topography SU-8 formed by $20 \mathrm{~nm}$ sub-pillars clearly showed superhydrophobicity (contact angles $>157^{\circ}$, contact angle hysteresis as low as $5^{\circ}$ ). Although there was a potential, optical transparency of the dual-scale topography of this SU-8 film was not reported.

We reported a method to create optically transparent nano-patterned SU-8 micro-pillar array as a superhydrophobic thin film [35]. As shown in Figure 10, a dense array of tapered SU-8 micro-pillar array was fabricated using previously reported SU-8 backside exposure method [36]. Then, it was isotropically dry etched for $10 \mathrm{~min}$. using $\mathrm{O}_{2} / \mathrm{CF}_{4}(90 \%$ : $10 \%)$ plasma with a power of $300 \mathrm{~W}$ in a microwave plasma etcher (TePla PS 300, PVA TePla America, Inc., Corona, CA, USA). The top and bottom diameter of the SU-8 micro-pillar was $50 \mu \mathrm{m}$ and $85 \mu \mathrm{m}$, respectively, and the height was $250 \mu \mathrm{m}$ with a $3^{\circ} \sim 5^{\circ}$ tapered angle (Figure 11a and b). Isotropic plasma etching process made nano-porous patterns $(10 \sim 900 \mathrm{~nm})$ uniformly on the surface of the non-planar micro-pillar structures (Figure 11c and d). The advancing angle of the this super-hydrophobic SU-8 thin film was in the range of $156^{\circ} \sim 161^{\circ}(0.025 \mu \mathrm{L} / \mathrm{sec}$. dispense rate $)$ and the receding angle was measured to be $122^{\circ} \sim 127^{\circ}$ (Figure 12). Optical transparency was found to be $60 \sim 70 \%$ by an n\&k analyzer which shows feasibility of the optically transparent applications (Figure 13).

\section{PDMS based super-hydrophobic thin film}

Although SU-8-based artificial super-hydrophobic thin films overall showed excellent hydrophobicity, the optical transparency in visible wavelengths turned out to be less than $80 \%$. For practical optically transparent self-cleaning thin film applications, it is highly desirable to have greater than $80 \%$ optical transparency. Polydimethylsiloxane (PDMS) was one of the popular materials studied by various groups for optically transparent artificial self-cleaning thin film applications. The popularity for the PDMS was due to many favorable inherent material properties. Some of the key material properties of the PDMS for the optically transparent super-hydrophobic thin film applications are its low surface energy of $19.8 \mathrm{~mJ} / \mathrm{cm}^{2}$ [37], high optical transparency throughout the ultraviolet and visible wavelengths [38], and extremely low Young's modulus ( $<4 \mathrm{MPa}$ ) [39] which makes the PDMS ideal material for flexible self-cleaning super-hydrophobic thin film application to conform to arbitrary shaped non-planar surfaces. It is also well-known for reliably maintaining dimensions in the replicated inverse images of a master mold using soft lithography [40].

Huang et al. demonstrated a dense array of replicated PDMS micro lens and PDMS micro bowls [41] by single and double soft lithography, respectively from an array of tall and sharp photoresist spikes using their unique 3D diffuser lithography technique [42]. The PDMS micro bowl array showed water contact angle of approximately $164.6^{\circ}$ and low adhesive force, outstanding characteristic for the self-cleaning thin film applications. Im et al. further demonstrated a robust super-hydrophobic and super-oleophobic surface with inverse trapezoidal microstructures on PDMS [43]. Again, they used their unique backside 3D diffuser lithography to fabricate a photoresist mold and PDMS trapezoidal structures were replicated using soft lithography method (Figure 14). The inverse transparent and flexible PDMS trapezoidal microstructures have the following dimensions: pitch distance of $40 \mu \mathrm{m}$, diameter of $26 \mu \mathrm{m}$ at the top, diameter of $15 \mu \mathrm{m}$ at the bottom. The contact angle of Teflon coated PDMS trapezoid surface was as high as $153^{\circ}$ and it also showed the high contact angle of $135^{\circ}$ using methanol droplet (Figure 15). 


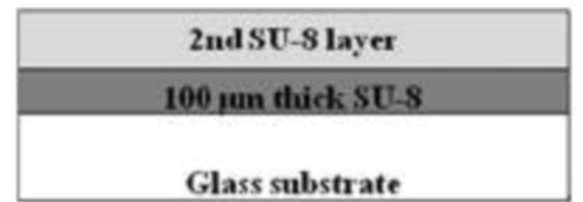

(a) Spin coating $(250 \mu \mathrm{m})$

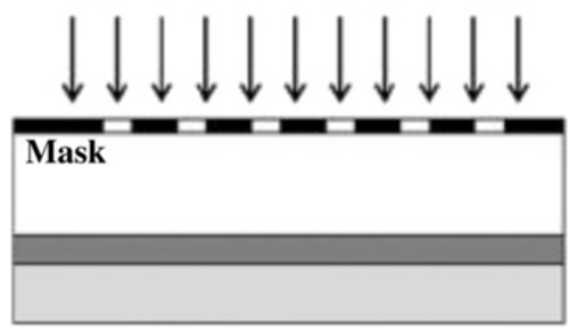

(b) Backside exposure

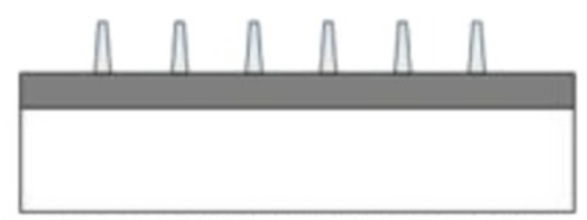

(c) Tapered pillar array formation

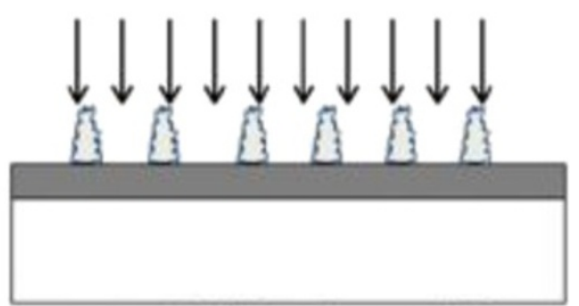

(d) $\mathrm{O}_{2} / \mathrm{CF}_{4}$ plasma etch

Figure 10 Fabrication sequence of the nano-patterned SU-8 micro-pillar array. (a) spin coating, (b) backside exposure, (c) Tapered pillar array formation, and (d) $\mathrm{O}_{2} / \mathrm{CF}_{4}$ plasma etch.

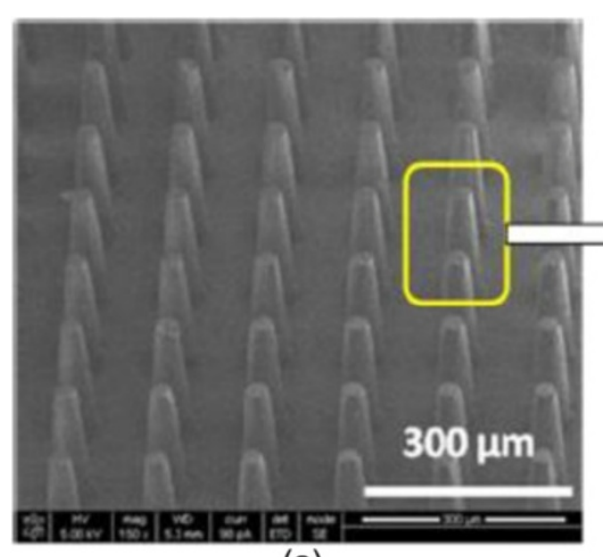

(a)

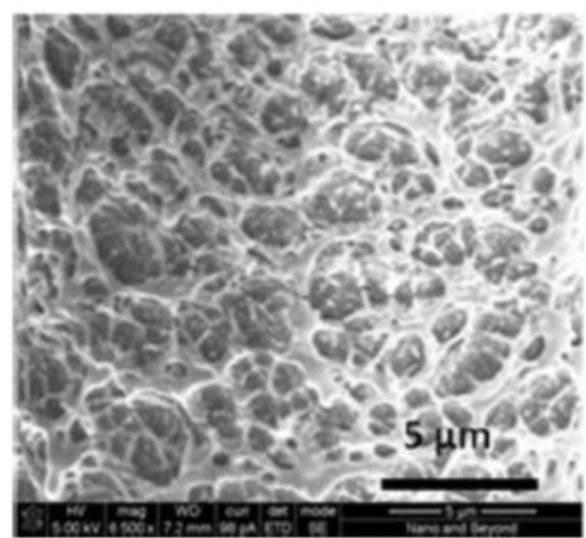

(c)

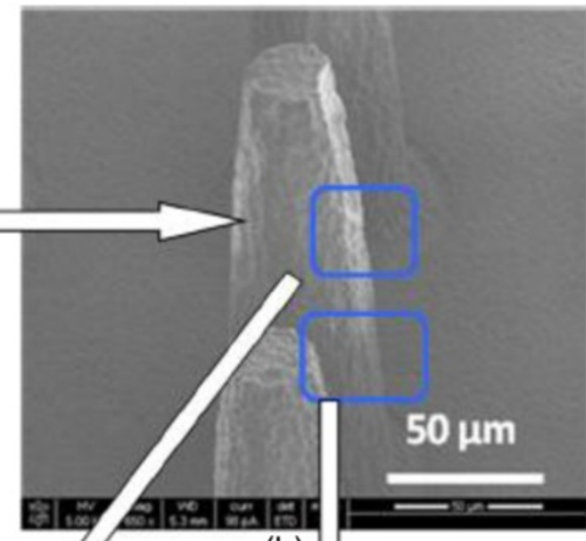

(b)

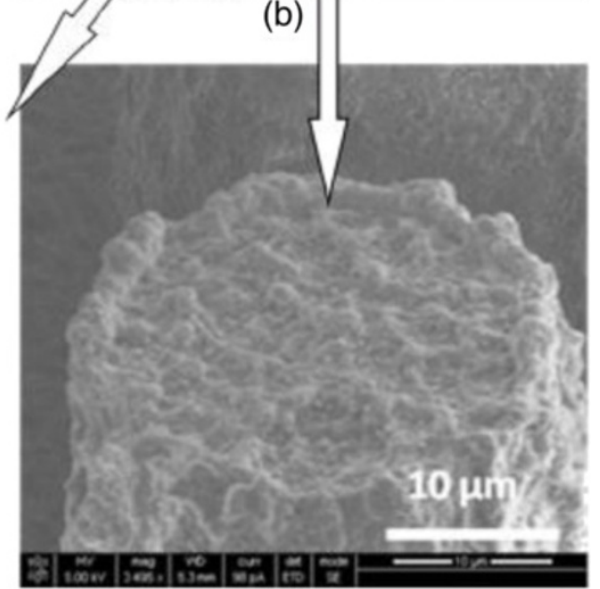

(d)

Figure 11 SEM images of nano-patterned SU-8 micro-pillar array: (a) after plasma treatment, (b) close-up view, (c) nano-patterned surface, and (d) plasma treated top pillar area. With permission from Journal of Micromechanic Microengineering, Copyright 2012. 


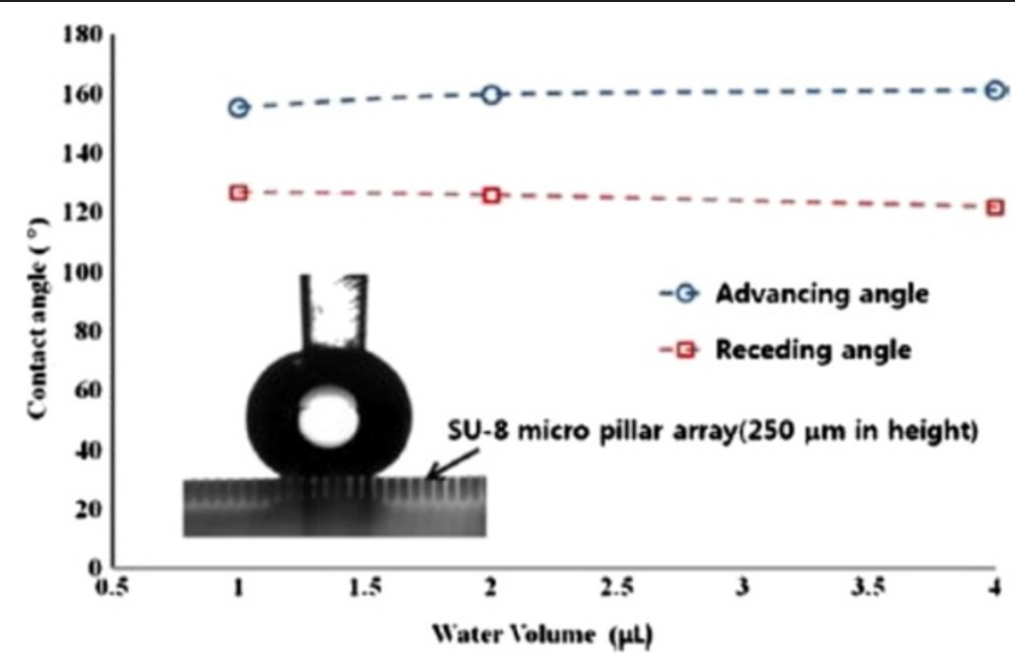

Figure 12 Dynamic contact angles of the SU-8 super-hydrophobic thin film. With permission from Journal of Micromechanic Microengineering, Copyright 2012.

Recently we reported an extremely simple one-step fabrication method of flexible, optically transparent superhydrophobic PDMS thin film using under-exposed underbaked photoresist mold [44]. As shown in Figure 16, this approach intentionally utilizes significant under soft baking so it allows photoresist retains good amount of solvent which in turn greatly increases the dissolution rate of the photoresist during the developing process. Once this intentional under baking condition is combined with under exposure condition, it would create a unique broccoli like structure with near randomly created nano-scale surface due to dissolved solvents. A positive PR, AZP4620 was severely under soft baked in an $88^{\circ} \mathrm{C}$ convection oven and subsequently it was under exposed with the exposure dose of $800 \mathrm{~mJ} / \mathrm{cm}^{2}$. After the photoresist was developed by AZ400K (1:3) developer, broccoli like micro-/nano-scale combined hierarchical structures was formed inside of the photoresist mold. Then, it was replicated by PDMS in a vacuum oven to completely replicate the extremely concave area on the surface of the photoresist. After the curing of PDMS, the photoresist mold was stripped away using AZ $400 \mathrm{~T}$ to safely delaminate the replicated PDMS from the mold. Figure 17 shows SEM images of natural lotus leaf compared with replicated PDMS super-hydrophobic surfaces prepared by this approach. As shown in Figure 18, the water static contact angle was $157.7 \pm 1^{\circ}, 160 \pm 1^{\circ}$, and $161 \pm 0.5^{\circ}$ for 4,10 , and $15 \mu \mathrm{L}$ droplets, respectively which clearly shows super-hydrophobic nature of the PDMS thin

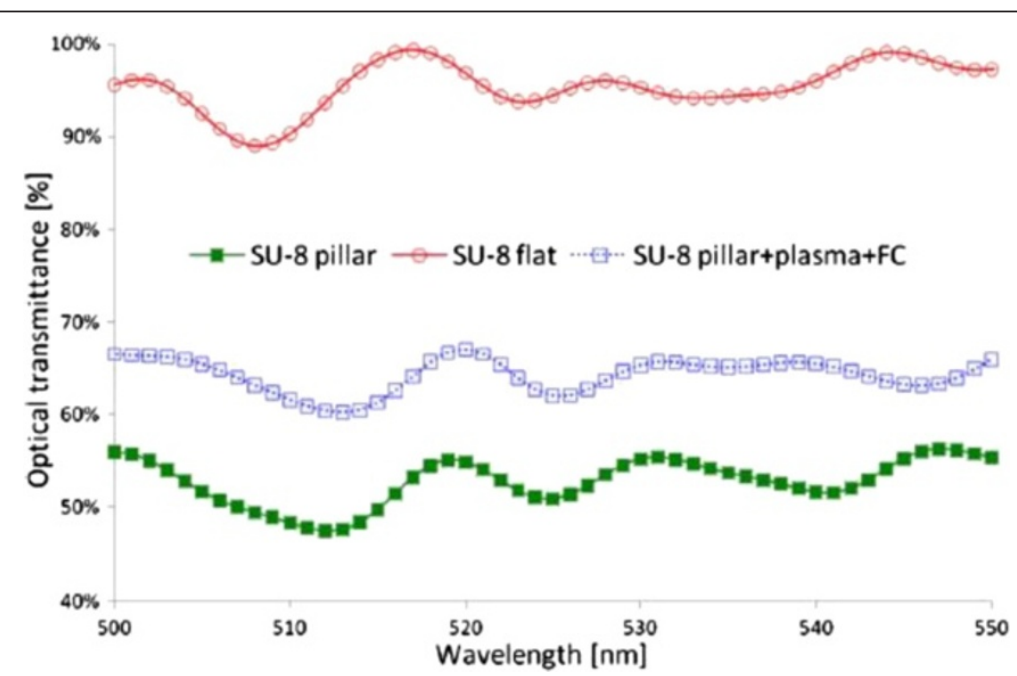

Figure 13 Optical transmittance for various films on the SU-8 surface. With permission from Journal of Micromechanic Microengineering, Copyright 2012. 


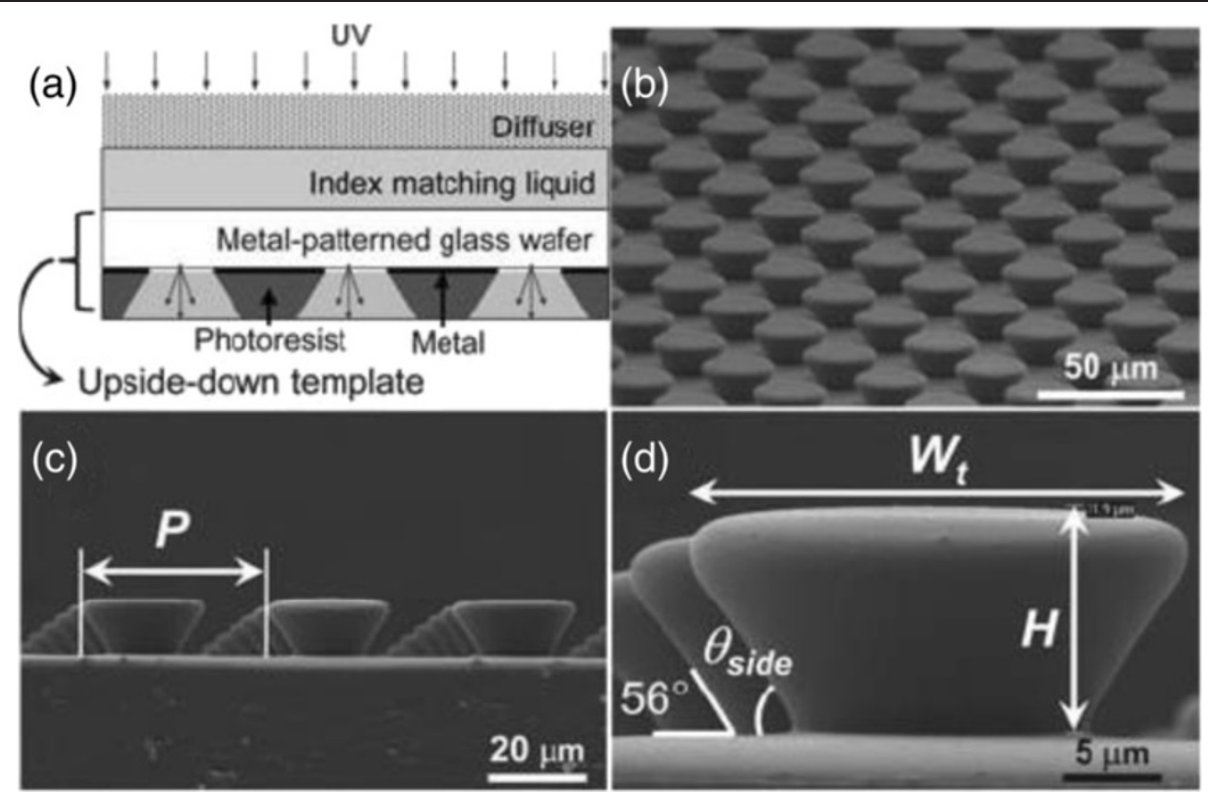

Figure 14 Unique backside 3D diffuser lithography. (a) A schematic diagram of backside 3D diffuser lithography, (b) SEM image of inverse trapezoid with magnification $(50 \mu \mathrm{m})$, (c) with magnification $(20 \mu \mathrm{m})$, (d) with magnification $(5 \mu \mathrm{m})$.

film. The water contact angles were slightly increased to the range of $160^{\circ} \sim 163^{\circ}$ by depositing $100 \mathrm{~nm}$ fluorocarbon $\left(\mathrm{C}_{\mathrm{x}} \mathrm{F}_{\mathrm{y}}\right)$ film. The optical transmittance of bare PDMS film was in the range of $95 \sim 100 \%$, while that of the broccolishaped super-hydrophobic PDMS film was in the range of 94 $~ 98 \%$ and the fluorocarbon-coated super-hydrophobic
PDMS film was in the range of $88 \sim 92 \%$ at around $550 \mathrm{~nm}$ wavelength [45]. As a demonstration of an application, Figure 19 shows an optical image of a solar panel covered with a super-hydrophobic PDMS film in which two water droplets were placed to show self-cleaning effect.

\section{(a)}

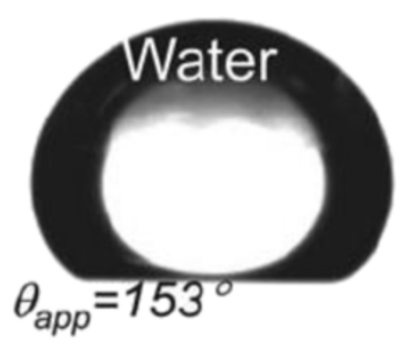

(b)
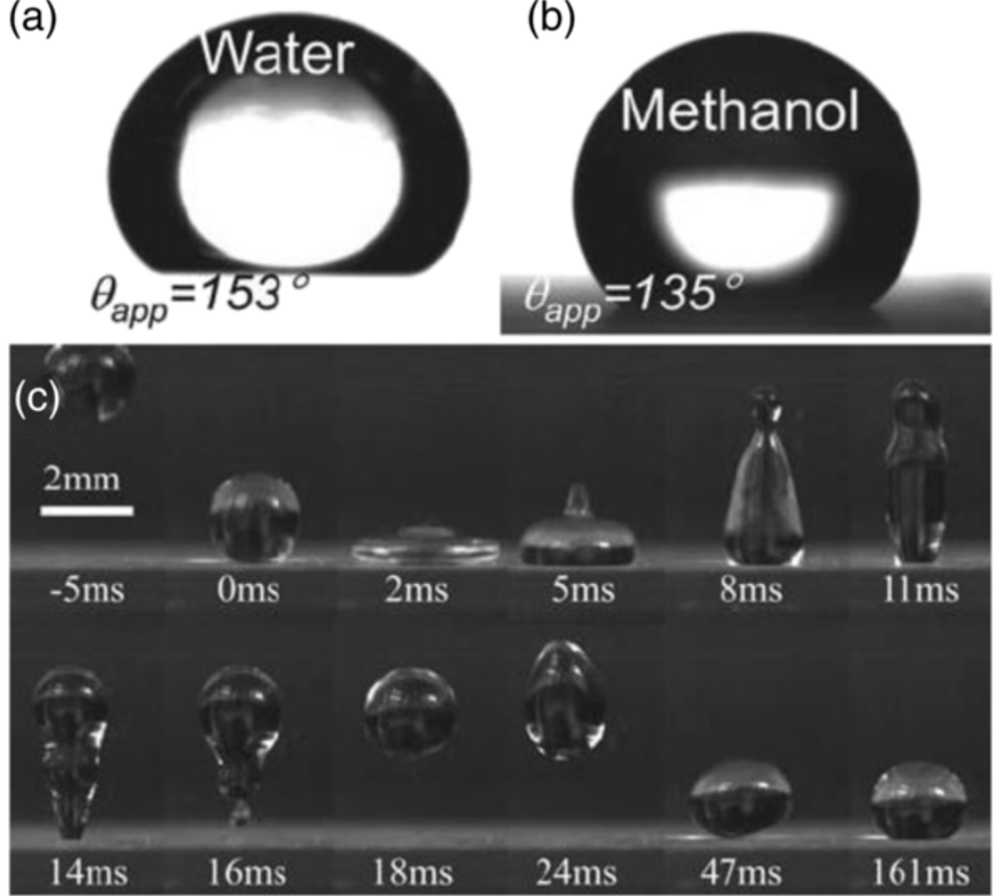

Figure 15 Super-hydrophobic characterizations. (a) Contact angle of Teflon coated trapezoid, (b) contact angle of the methanol, and (c) snapshots of high speed camera images of bouncing water droplet. 


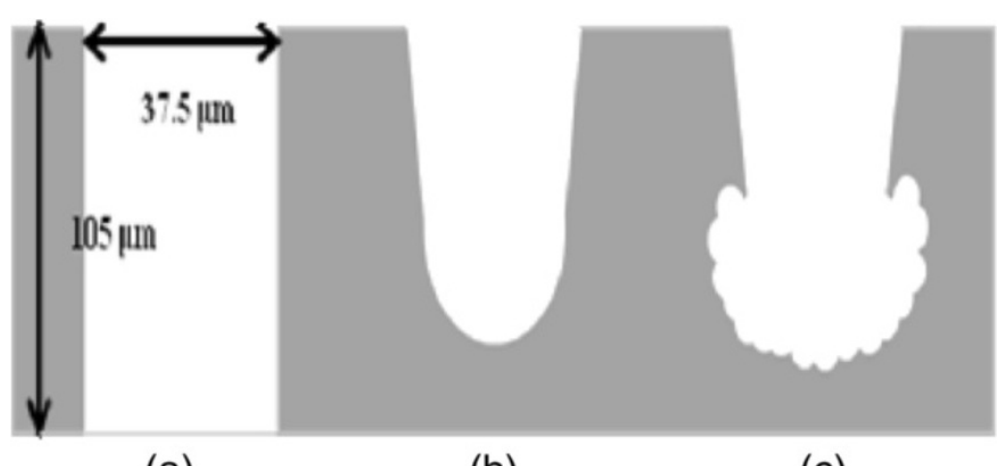

(a)

(b)

(c)

Figure 16 Schematic diagrams of the photoresist patterning: (a) conventional lithography, (b) under-exposure condition, and (c) underexposure under-baking condition. With permission from IEEE MEMS, Copyright 2012.

\section{Super-lyophobic surfaces against liquid metals}

Liquid metal is the metal in liquid phase at room temperature. As it has liquid property which is continuously deformable as well as metallic property such as high thermal and electrical conductivity, it can be applied to various applications such as radio frequency (RF) MEMS switch [46], electro-wetting on dielectrics (EWOD) [47,48], tunable and flexible antennas [49], frequency selective surfaces (FSS) [50], and heat transfer [51] among others. For those various applications, as the surface oxidation of liquid metal drastically increases the wettability, the superlyophobic surface against liquid metal has been of interest.

\section{Super-lyophobic surfaces against mercury}

The most well-known liquid metal is mercury. The surface tension and contact angle of various substrates against mercury have been studied from 1920s. The reported surface tension of mercury is in the range of $400 \sim 516 \mathrm{mN} / \mathrm{m}$ [52] in air environment at room temperature. The typically acceptable value of surface tension is $\sim 480 \mathrm{mN} / \mathrm{m}$ [53] which is much greater than that of water $(72.9 \mathrm{mN} / \mathrm{m})$. Therefore, the contact angle of mercury is much higher than that of water on the same solid substrate based on Young's equation [9].

As the contact angle study was investigated without development of micromachining technology, most studied substrates were simply flat surface but treated with different chemicals such as low surface energy material like Teflon (surface energy $\sim 18.5 \mathrm{mN} / \mathrm{m}$ ). Yarnold reported that the advancing and receding contact angle of mercury on the steel which was washed in ether were $165^{\circ}$ and $130^{\circ}$, respectively [54]. Gray also reported dynamic contact angle of mercury on low-energy solid substrates such as polyethylene, paraffin wax, and PTFE. The highest advancing angle of $151.7^{\circ}$ was obtained on the PTFE substrate and the lowest contact angle hysteresis of $\sim 0.2^{\circ}$ was achieved on the paraffin wax with pure mercury by acid treatment [55]. Ellison et al. studied the dynamic contact angles of mercury on various substrates (tungsten, stainless steel, nickel, quartz, glass, and Teflon) under varying temperatures in the range of $25 \sim 150^{\circ} \mathrm{C}$ [56]. The highest advancing contact angle of $157^{\circ}$ was measured on the Teflon as the Teflon is 'low surface energy' substrate compared with other 'high surface energy' substrates. In addition, most substrates showed that contact angle hystereses were in the range of $0^{\circ} \sim 3^{\circ}$ with pure cleaned mercury which means that the mercury can be easily roll off from the surface. The temperature effect for surface tension of mercury was negligible. Awasthi et al. reported the static contact angle of mercury on graphite as $152.5 \pm 2^{\circ}$ by applying 'spot technique' which can be also used for high temperature measurement, as the contact angle of liquid metal at high temperature is useful for practical applications such as metal casting, welding, and brazing [57].

In particular, the contact angle of mercury on the dielectric substrate was an important parameter for EWOD applications $[47,48]$. Shen et al. reported that the contact angle of line patterns with various contact ratio (line width per pitch) for mercury using photolithography [47]. As the contact ratio decreases from 1 to 0.3 , the contact angle increases from $142^{\circ}$ to $158^{\circ}$. Latorre et al. reported that the contact angle of mercury on oxidized silicon wafer was $137^{\circ}$ with a standard deviation of $8^{\circ}$ [48].

Recently, super-mercury-phobic surface with hierarchical structures was demonstrated by Escobar et al. [58]. The thermal oxidation creates pyramids with sub-micron protrusions resulting in hierarchical structures with micro/ nano structures as shown in Figure 20. The contact angle of boron doped diamond surfaces with thermal oxidation is greater than $175^{\circ}$ with no contact angle hysteresis (Figure 21).

\section{Super-lyophobic surfaces against gallium-based liquid metal alloys}

Non-toxic gallium-based liquid metal have been of interest lately as it has various favorable properties such as higher 


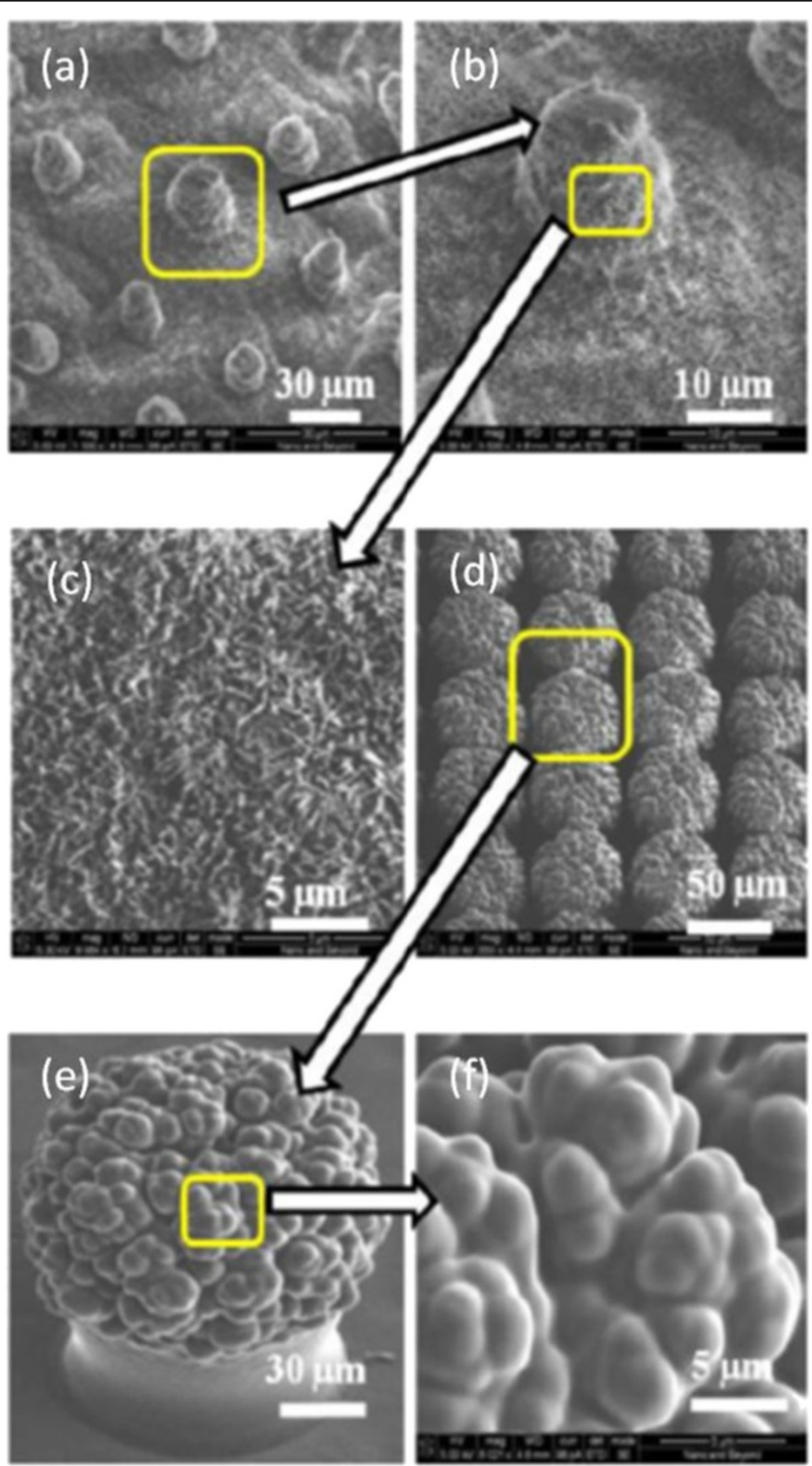

Figure 17 SEM images of super-hydrophobic surface: (a-c) natural lotus leaf and (d-f) PDMS artificial super-hydrophobic surface. With permission from IEEE MEMS, Copyright 2012.

boiling point, higher thermal and electrical conductivity against mercury [59]. The gallium-based binary (eutectic GaIn alloy [8]) and ternary alloy (e.g., Galinstan $\left.{ }^{\circ}[59]\right)$ have been studied for various applications. However, it has a challenging problem which is the fact that the surface of gallium-based liquid metal alloy is instantly oxidized in air and wets almost any surfaces [60]. It is a critical issue to have non-wetting super-lyophobic surface against galliumbased liquid metal but there was very limited report on super-lyophobic surface for gallium-based liquid metal alloys, probably due to the strong wettability of the galliumbased alloys.

In order to prevent of wetting, Liu et al. measured, in the below $1 \mathrm{ppm}$ oxygen environment, the advancing and 


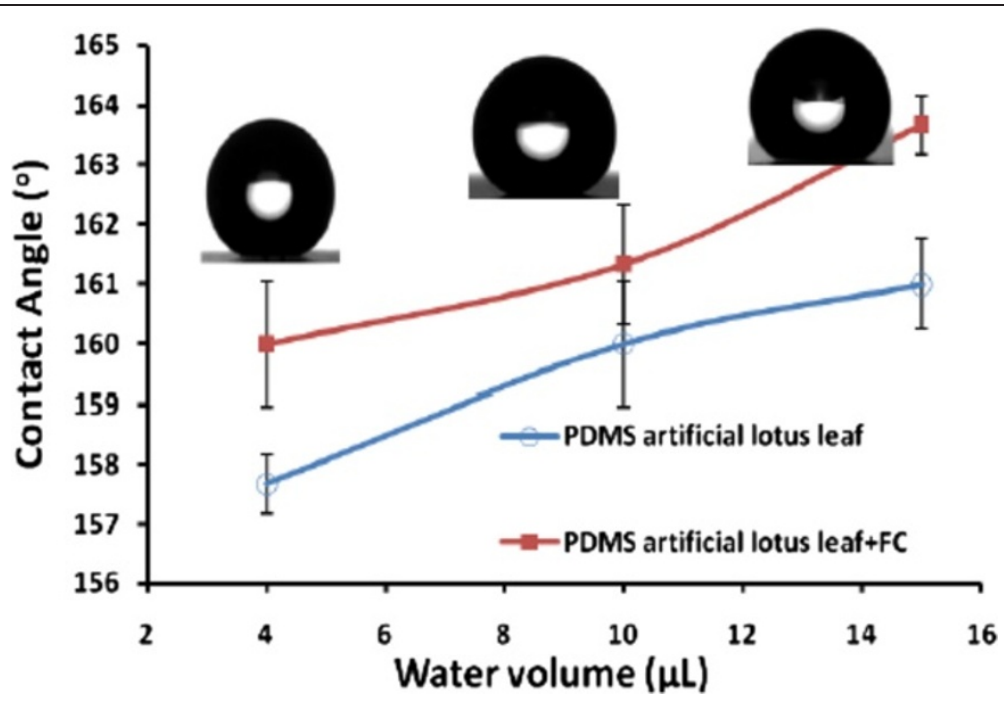

Figure 18 Water static contact angles of the artificial super-hydrophobic surfaces. With permission from IEEE MEMS, Copyright 2012.

receding contact angle of pure Galinstan ${ }^{\circ}$ on the various substrates such as tungsten, silicon nitride, glass, parylene, Teflon $^{\circ}$, phlogopite, and muscovite. It was found that Galinstan $^{\circ}$ was non-wettable on all surfaces under the condition. Among them, the muscovite showed the highest advancing of $163.6^{\circ}$ and receding contact angle of $148.1^{\circ}$, resulting in the smallest contact angle hysteresis of $15.5^{\circ}$, as the substrate has higher surface roughness compared to others [59].

Oxide-free gallium-based liquid metal alloys are crucial for certain applications like micro switches. However, in other applications such as micro-cooling and FSS, maintaining true liquid phase of gallium-based liquid metal alloy is not necessary. Recently, we measured static contact angle of oxidized Galinstan ${ }^{\bullet}$ on a glass, Cytop, and
Teflon, in air environment [61]. Among them, Teflon substrate shows highest contact angle of $140.3^{\circ}$. However, the contact angle of the Galinstan ${ }^{\circ}$ droplet was increased to be $152.5^{\circ}$ by removing oxide layer using hydrochloric acid vapor.

In addition, we tested efficacy of the hierarchical micro-/nano-scale super-hydrophobic thin film formation technique to gallium-based liquid metal alloy. With under-baked and under-exposed photoresist mold as explained in section 3.2.4, PDMS multi-scale surface extured micro pillar array was fabricated. We found the Cassie and Wenzel states as well as transition state as shown in Figure 22 [62]. In addition, we studied lyophobicity of various pitch distance in the range of $50 \sim 525 \mu \mathrm{m}$ micro-/nano-scale hierarchical micro pillar

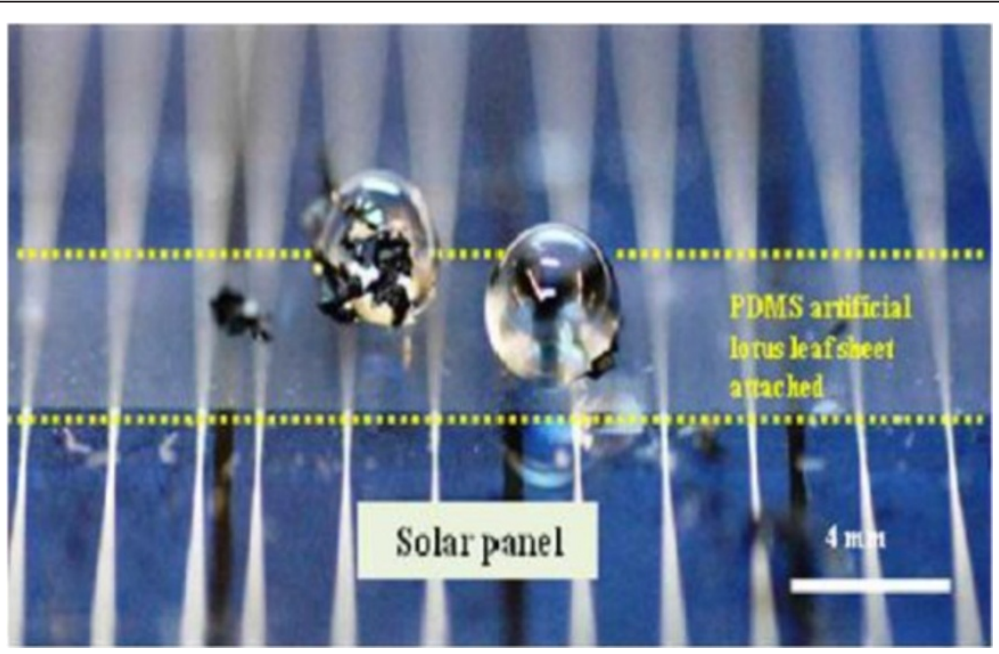

Figure 19 Optical images of $10 \mu \mathrm{L}$ water droplet on the broccoli like artificial super-hydrophobic thin film. With permission from IEEE MEMS, Copyright 2012. 


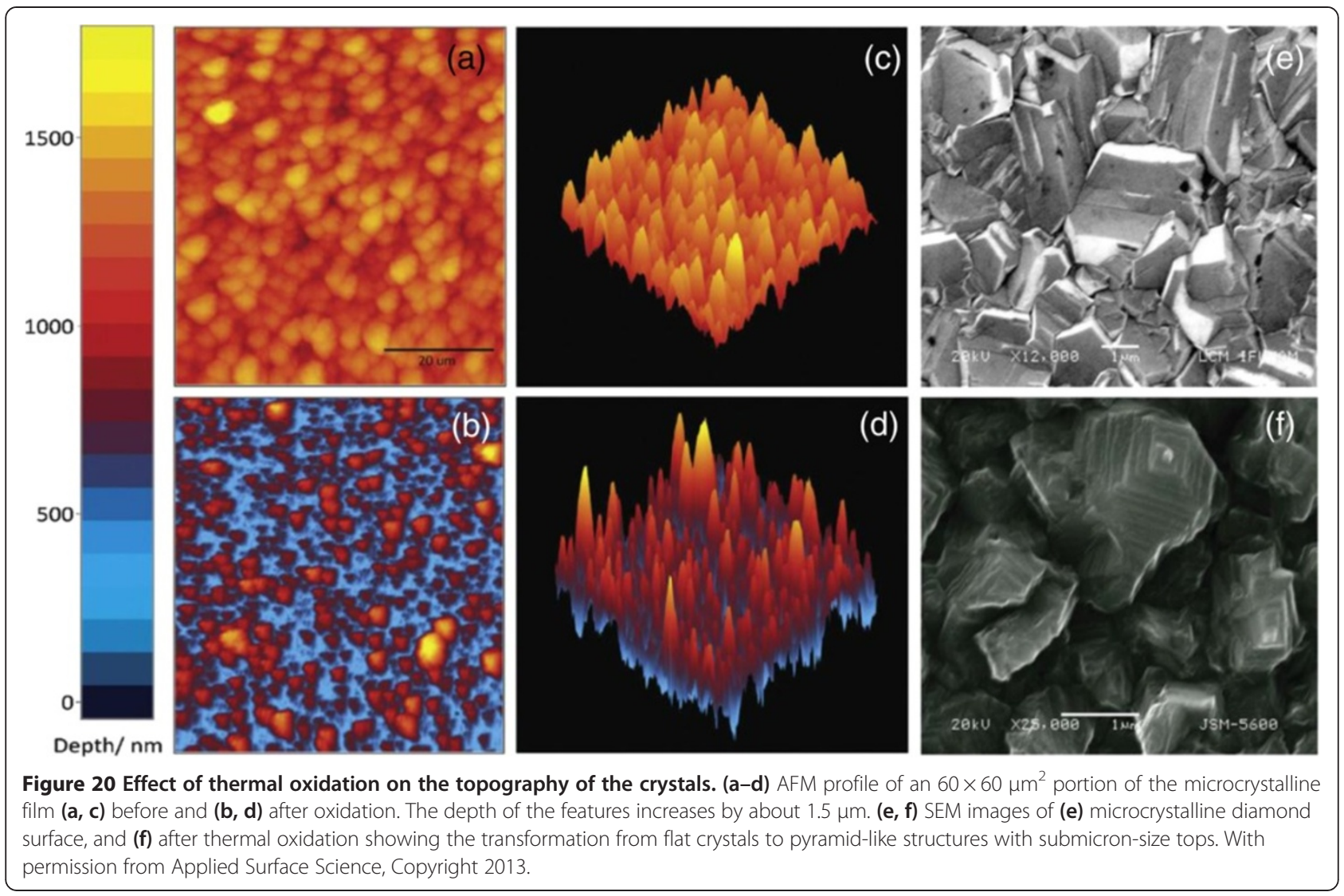

array by measuring contact and sliding angels (Figure 23). By conjunction with study of contact images on various pitch distance micro pillar arrays, the contact angles of micro pillar arrays with pitch distance $<275 \mu \mathrm{m}$ (in Cassie state) were higher than those of the micro pillar arrays with pitch distance $>275 \mu \mathrm{m}$ (in Wenzel state). In addition, the sliding angle could be only obtained on micro pillar arrays with pitch distance $<275 \mu \mathrm{m}$ (in Cassie state). The highest static contact angle of $163^{\circ}$ and the lowest sliding angle of $17.4^{\circ}$ were achieved on the $175 \mu \mathrm{m}$
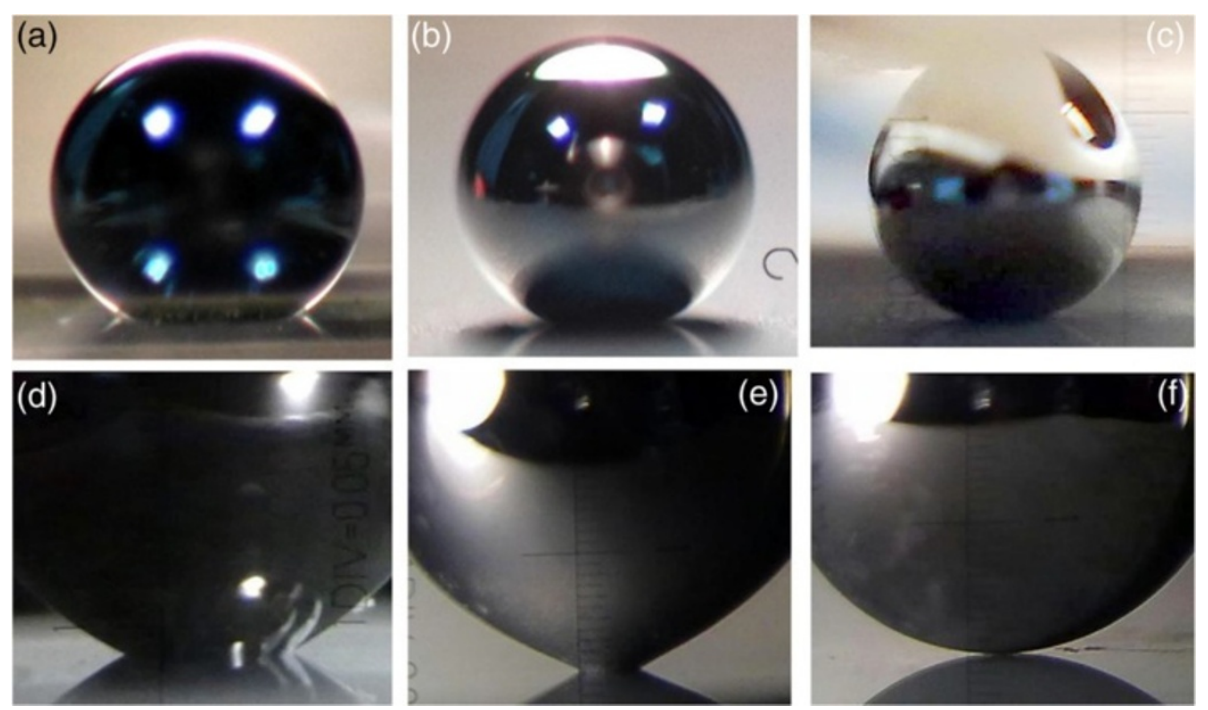

Figure 21 Contact angle of mercury droplet. Contact between droplets of mercury resting on the surfaces $(\mathbf{a}, \mathbf{b}, \mathbf{c})$ and being detached from them (d, e, f). The columns correspond to $(\mathbf{a}, \mathbf{d})$ the polished singe crystal, $(\mathbf{b}, \mathbf{e})$ the micro-crystalline diamond and $(\mathbf{c}, \mathbf{f})$ the oxidized sample. 
(a)

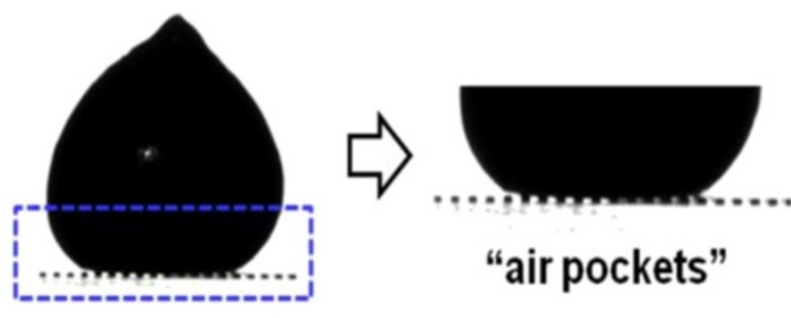

(b)

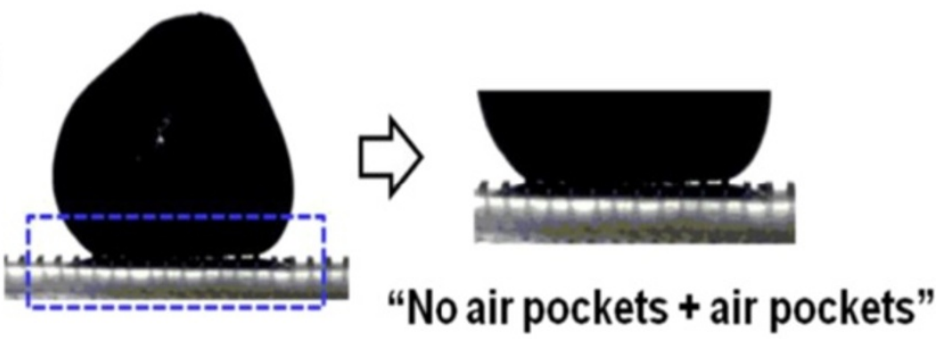

(c)

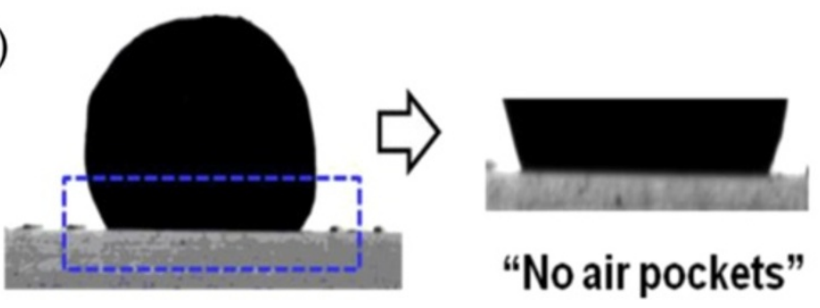

Figure $227.8 \mu \mathrm{L}$ oxidized Galinstan ${ }^{\otimes}$ droplet on (a) $175 \mu \mathrm{m}$ pitch pillar array, forming a non-wetting Cassie state (air pockets), (b) $275 \mu \mathrm{m}$ pitch pillar array, on which the droplet partially fill in surface texture (air pockets and no air pockets area), and (c) $525 \mu \mathrm{m}$ pitch pillar array (no air pockets), where the droplet fully wets the surface texture. With permission from IEEE MEMS, Copyright 2012.

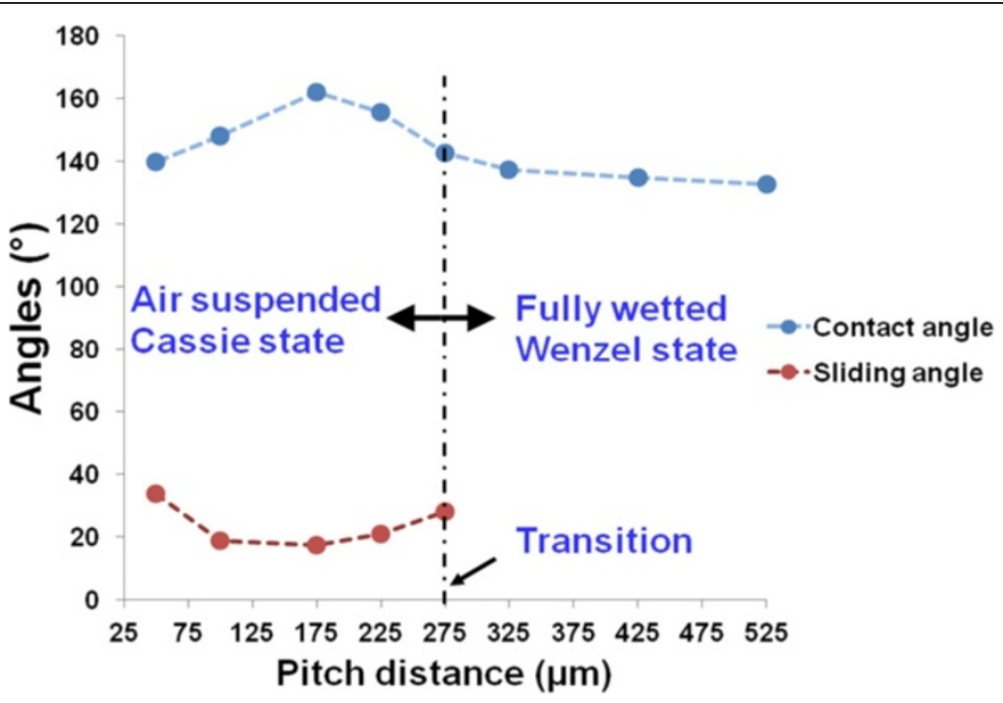

Figure 23 Contact and sliding angle as a function of pitch distance for $7.8 \mu \mathrm{L}$ oxidized Galinstan ${ }^{\circledR}$ droplet. With permission from IEEE MEMS, Copyright 2012. 
pitch distance pillar array. The result clearly showed that the high contact angle and low sliding angle were achieved due to micro-/nano-scale combined hierarchical textures with an optimum pitch distance.

\section{Conclusions}

Recent progress on the hierarchical micro-/nano-scale structures for the artificial super-hydrophobic surfaces and super-lyophobic surfaces against gallium-based liquid metal alloys are reviewed. As one of the key contributing factors for the natural super-hydrophobic surfaces is dual micro-/ nano-scale surfaces, a wide variety of materials and numerous fabrication approaches were studied. Of those, optically transparent artificial super-hydrophobic surfaces are of great interest in many practical self-cleaning surface applications. Both SU-8 and PDMS-based super-hydrophobic self-cleaning surfaces have been demonstrated with promising characteristics. As demonstrated by a few groups, it is in its utmost importance to realize super-hydrophobic and super-oleophobic surfaces together to be used in real applications.

Gallium-based liquid metal alloys are one of the very interesting materials and have great potential to be utilized in a wide variety of novel applications due to their unique conductive characteristic along with constantly deformable liquid characteristic. Due to its oxidation problem, it is essential to have super-lyophobic surface against the oxidized gallium-based liquid metal. Recently hierarchical micro-/nano-scale structures have been applied to superlyophobicity surfaces against gallium-based liquid metals. It is expected that, in the next few years, numerous unexplored potential of the liquid metal-based devices will be studied based on recent progress of the super-lyophobic surfaces.

\section{Competing interests}

The authors declare that they have no competing interests.

\section{Authors' contributions}

YY carried out research works and a survey on the super-hydrophobic surfaces. DK carried out research works and a survey on the liquid metals. JL supervised all research works and writing of the manuscript. All authors read and approved the final manuscript.

\section{Acknowledgement}

The authors would like to thank Dr. Dong-Weon Lee of Chonnam National University and Dr. Wonjae Choi of The University of Texas at Dallas for invaluable technical/scientific discussions and UTD clean room staff for their support on device fabrication work. The authors also would like to thank Republic of Korea (ROK) Army for financial support.

Received: 13 November 2013 Accepted: 27 May 2014

Published online: 03 September 2014

\section{References}

1. Smith J, Jones M Jr, Houghton L (1999) Future of health insurance. N Engl J Med 965:325-329

2. Byun D, Hong J, Saputra KJ, Lee Y, Park H, Byun B, Lukes J (2009) Wetting characteristics of insect wing surfaces. J Bionic Eng 6:63-70
3. Feng L, Li S, Li Y, Li H, Zhang L, Zhai J, Song Y, Liu B, Jiang L, Zhu D (2002) Superhydrophobic surfaces: from natural to artificial. Adv Materials 14:1857-1860

4. Koch K, Barthlott W (2009) Superhydrophobic and superhydrophilic plant surfaces: an inspiration for biomimetic materials. Phil Trans Royal Soc A: Math Phys Eng Sci 367:1487-1509

5. Bhushan B, Jung $Y$ (2008) Wetting, adhesion and friction of superhydrophobic and hydrophilic leaves and fabricated micro/nanopatterned surfaces. J Phys: Cond Matter 20:225010

6. Nakajima A, Fujishima A, Hashimoto K, Watanabe T (1999) Preparation of transparent superhydrophobic boehmite and silica films by sublimation of aluminum acetylacetonate. Adv Materials 11:1365-1368

7. Hoefnagels H, Wu D, With G, Ming W (2007) Biomimetic superhydrophobic and highly oleophobic cottont textiles. Langmuir 23:13158-13163

8. Dickey M, Chiechi R, Larsen R, Weiss E, Weitz D, Whitesides G (2008) Eutectic gallium-indium (EGaln): a liquid metal alloy for the formation of stable structures in microchannels at room temperature. Adv Func Materials 18:1097-1104

9. Young T (1805) An essay on the cohesion of fluids. Phil Trans Royal Soc London 95:65-87

10. Wenzel R (1936) Resistance of solid surfaces to wetting by water. Ind Eng Chem 28:988-994

11. Baxter S, Cassie A (1945) 8-The water repellency of fabrics and a new water repellecncy test. J Textile Ins Trans 36:T67-T90

12. Choi W, Tuteja A, Mabry J, Cohen R, McKinley G (2009) A modified Cassie-Baxter relationship to explain contact angle hysteresis and anisotropy on non-wetting textured surfaces. J Colloid Int Sci 339:208-216

13. Hozumi A, McCarthy T (2009) Ultralyophobic oxidized aluminum surfaces exhibiting negligible contact angle hysteresis. Langmuir 26:2567-2573

14. Barthlott W, Neinhuis C (1997) Purity of the sacred lotus, or escape from contamination in biological surfaces. Planta 202:1-8

15. Burton Z, Bhushan B (2006) Surface characterization and adhesion and friction properties of hydrophobic leaf surfaces. Ultramicroscopy 106:709-719

16. Bhushan B, Jung Y, Koch K (2009) Micro-, nano- and hierarchical structures for superhydrophobicity, self-cleaning and low adhesion. Phil Trans Royal Soc A Math Phys Eng Sci 367:1631-1672

17. Guo Z, Liu W (2007) Biomimic from the superhydrophobic plant leaves in nature: binary structure and unitary structure. Plant Sci 172:1103-1112

18. Cha T, Yi J, Moon M, Lee K, Kim H (2010) Nanoscale patterning of microtextured surfaces to control superhydrophobic robustness. Langmuir 26:8319-8326

19. Kang C, Lee S, Jung I, Jung P, Hwang S, Ko J (2008) The fabrication of patternable silicon nanotips using deep reactive ion etching. J Micromech Microeng 18:075007

20. Roach P, Shirtcliffe N, Newton M (2008) Progess in superhydrophobic surface development. Soft Matter 4:224-240

21. Cortese B, D'Amone S, Manca M, Viola I, Cingolani R, Gigli G (2008) Superhydrophobicity due to the hierarchical scale roughness of PDMS surfaces. Langmuir 24:2712-2718

22. Wang M, Raghunathan N, Ziaie B (2007) A nonlithographic top-down electrochemical approach for creating hierarchical (micro - nano) superhydrophobic silicon surfaces. Langmuir 23:2300-2303

23. Bhushan B, Jung Y, Niemietz A, Koch K (2009) Lotus-like biomimetic hierarchical structures developed by the self-assembly of tubular plant waxes. Langmuir 25:1659-1666

24. Ming W, Wu D, Benthem R, With G (2005) Superhydrophobic films from raspberry-like particles. Nano Lett 5:2298-2301

25. Bok H, Kim S, Yoo S, Kim S, Park S (2008) Synthesis of perpendicular nanorod arrays with hierarchical architecture and water slipping superhydrophobic properties. Langmuir 24:4168-4173

26. Li H, Wang X, Song Y, Liu Y, Li Q, Jiang L, Zhu D (2003) Super-amphiphobic aligned carbon nanotube films. J Am Chem Soc 40:1743-1746

27. Lin J, Chu C, Chiang M, Tsai W (2006) Manipulating assemblies of high-aspectratio clays and fatty amine salts to form surfaces exhibiting a lotus effect. Adv Materials 18:3248-3252

28. Stöber W, Fink A, Bohn E (1968) Controlled growth of monodisperse silica spheres in the micron size range. J Colloid Int Sci 26:62-69

29. Qian Z, Zhang Z, Song L, Liu H (2009) A novel approach to raspberrylike particles for superhydrophobic materials. J Materials Chem 19:1297-1304

30. Lau K, Bico J, Teo K, Chhowalla M, Amaratunga G, Milne W, McKinley G, Gleason K (2003) Superhydrophobic carbon nanotube forests. Nano Lett 3:1701-1705 
31. Jung $Y$, Bhushan B (2009) Mechanically durable carbon nanotube-composite hierarchical structures with superhydrophobicity, self-cleaning, and low-drag. ACS Nano 3:4155-4163

32. Zhang J, Zhou W, Chan-Park M, Conner S (2005) Argon plasma modification of SU-8 for very high aspect ratio and dense copper electroforming. J Electrochem Soc 152:C716-C721

33. Hong L, Pan T (2010) Photopatternable superhydrophobic nanocomposites for microfabrication. J Microelectromech Sys 19:246-253

34. Marquez-Velasco J, Vlachopoulou M, Tserepi A, Gogolides E (2010) Stable superhydrophobic surfaces induced by dual-scale topography on SU-8. Microelectronic Eng 87:782-785

35. Yoon Y, Lee D, Lee J (2012) Surface modified nano-patterned SU-8 pillar array optically transparent super-hydrophobic thin film. J Micromech Microeng 22:035012

36. Kim K, Park D, Lu H, Che W, Kim K, Lee J, Ahn C (2004) A tapered hollow metallic microneedle array using backside exposure of SU-8. J Micromech Microeng 14:597

37. Hur S, Khang D, Kocabas C, Rogers J (2004) Nanotransfer printing by use of noncovalent surface forces: applications to thin-film transistors that use single-walled carbon nanotube networks and semiconducting polymers. Appl Phys Lett 85:5730-5732

38. Jo B, Lerberghe L, Motsegood K, Beebe D (2000) Three-dimensional microchannel fabrication in polydimethylsiloxane (PDMS) elastomer. J Microelectromech Sys 9:76-81

39. Fuard D, Tzvetkova-Chevolleau T, Decossas S, Tracqui P, Schiavone P (2008) Optimization of poly-di-methyl-siloxane (PDMS) substrates for studying cellular adhesion and motility. Microelectronic Eng 85:1289-1293

40. Kim K, Park S, Lee J, Manohara H, Desta Y, Murphy M, Ahn C (2002) Rapid replication of polymeric and metallic high aspect ratio microstructures using PDMS and LIGA technology. Microsyst Tech 9:5-10

41. Huang X, Kim D, Im M, Lee J, Yoon J, Choi Y (2009) Lock-and-key geometry effect of patterned surfaces: wettability and switching of adhesive force. Small 5:90-94

42. Lee J, Choi W, Lee K, Yoon J (2008) A simple and effective fabrication method for various 3D microstructures: backside 3D diffuser lithography. J Micromech Microeng 18:125015

43. Im M, Im H, Lee J, Yoon J, Choi Y (2010) A robust superhydrophobic and superoleophobic surface with inverse-trapezoidal microstructures on a large transparent flexible substrate. Soft Matter 6:1401-1404

44. Yoon Y, Lee D, Ahn J, Sohn J, Lee J (2012) One-step fabrication of optically transparent polydimethylsiloxane artificial lotus leaf film using under-exposed under-baked photoresist mold. In: Proc. IEEE 25th International Conference on Micro Electro Mechanical Systems. IEEE MEMS Conference, Paris, France, pp 301-304

45. Yoon Y, Lee D, Lee J (2013) Fabrication of optically transparent PDMS artificial lotus leaf film using underexposed and underbaked photoresist mold. J Microelectromech Sys 22:1073-1080

46. Sen P, Kim C (2009) Microscale liquid-metal switches : a review. Trans Ind Elect 56:1314-1330

47. Shen W, Kim J, Kim C (2002) Controlling the adhesion force for electrostatic actuation of microscale mercury drop by physical surface modification. In: Proc. IEEE 15th International Conference on Micro Electro Mechanical Systems. IEEE MEMS Conference, Las Vegas, NV, USA, pp 52-55

48. Latorre L, Kim J, Lee J, Guzman P, Lee H, Nouet P, Kim C (2002) Electrostatic actuation of microscale liquid-metal droplets. J Microelectromech Sys 11:302-308

49. Kubo M, Li X, Kim C, Hashimoto M, Wiley B, Ham D, Whitesides G (2010) Stretchable microfluidic radiofrequency antennas. Adv Materials 22:2749-2752

50. Meng L, Bin Y, Behdad N (2010) Liquid-tunable frequency selective surfaces. Microwave Wireless Comp Lett 20:423-425

51. Ma K, Liu J (2007) Heat-driven liquid metal cooling device for the thermal management of a computer chip. J Phys D Appl Phys 40:4722

52. Nicholas M, Joyner $P$, Tessem B, Olson M (1961) The effect of various gases and vapors on the surface tension of mercury. J Phys Chem 65:1373-1375

53. Lide D (2007) CRC Handbook of Chemistry and Physics. CRC Press, Boca Raton, FL

54. Yarnold G (1946) The hysteresis of the angle of contact of mercury. Proc Phys Soc 58:120

55. Gray V (1966) Contact angles of mercury on low-energy solids. Nature 209:608-609

56. Ellison A, Klemm R, Schwartz A, Grubb L, Petrash D (1967) Contact angles of mercury on various surfaces and the effect of temperature. J Chem Eng Data 12:607-609
57. Awasthi A, Bhatt Y, Garg S (1996) Measurement of contact angle in systems involving liquid metals. Meas Sci Tech 7:753

58. Escobar J, Garza C, Alonso J, Castillo R (2013) Super-mercuryphobic and hydrophobic diamond surfaces with hierarchical structures: vanishment of the contact angle hysteresis with mercury. Appl Surf Sci 273:692-701

59. Liu T, Sen P, Kim C (2012) Characterization of nontoxic liquid-metal alloy Galinstan for applications in microdevices. J Microelectromech Sys 21:443-450

60. Scharmann F, Cherkashinin G, Breternitz V, Knedlik C, Hartung G, Weber T, Schaefer J (2004) Viscosity effect on GalnSn studied by XPS. Surf Int Anal 36:981-985

61. Kim D, Thissen P, Viner G, Lee D, Choi W, Chabal Y, Lee J (2013) Recovery of nonwetting characteristics by surface modification of gallium-based liquid metal droplets using hydrochloric acid vapor. ACS Appl Materials Int 5:179-185

62. Kim D, Lee D, Choi W, Lee J (2012) A super-lyophobic PDMS micro-tunnel as a novel microfluidic platform for oxidized Galinstan ${ }^{\oplus}$. In: Proc. IEEE 25th International Conference on Micro Electro Mechanical Systems. IEEE, Paris, France, pp 1005-1008

doi:10.1186/s40486-014-0003-x

Cite this article as: Yoon et al:: Hierarchical micro/nano structures for super-hydrophobic surfaces and super-lyophobic surface against liquid metal. Micro and Nano Systems Letters 2014 2:3.

\section{Submit your manuscript to a SpringerOpen ${ }^{\circ}$ journal and benefit from:}

- Convenient online submission

- Rigorous peer review

- Immediate publication on acceptance

- Open access: articles freely available online

- High visibility within the field

- Retaining the copyright to your article

Submit your next manuscript at $>$ springeropen.com 